Proceedings of SPIE, Vol. 5825, 118-138 (2005)

\title{
Ultrafast coherent spectroscopy of single semiconductor quantum dots
}

\author{
Christoph Lienau ${ }^{a}$, Thomas Unold ${ }^{a}$, Kerstin Mueller $^{a}$ and Thomas Elsaesser ${ }^{a}$ \\ ${ }^{a}$ Max-Born-Institut für Nichtlineare Optik und Kurzzeitspektroskopie, Max-Born-Straße 2A, \\ D-12489 Berlin, Germany;
}

\begin{abstract}
Excitonic and spin excitations of single semiconductor quantum dots currently attract attention as possible candidates for solid state based implementations of quantum logic devices. Due to their rather short decoherence times in the picosecond to nanosecond range, such implementations rely on using ultrafast optical pulses to probe and control coherent polarizations. In this article, we review our recent work on combining ultrafast spectroscopy and near-field microscopy to probe the nonlinear optical response of a single quantum dot and of a pair of dipolecoupled quantum dots on a femtosecond time scale. We demonstrate coherent control of both amplitude and phase of the coherent quantum dot polarization by studying Rabi oscillations and the optical Stark effect in an individual dot. By probing Rabi oscillations in a pair of dots, we identify couplings between permanent excitonic dipole moments. Our results show that although semiconductor quantum dots resemble in many respects atomic systems, Coulomb many-body interactions can contribute significantly to their optical nonlinearities on ultrashort time scales. This paves the way towards the realization of potentially scalable nonlocal quantum gates in chains of dipole-coupled dots, but also means that decoherence phenomena induced by many-body interactions need to be carefully controlled.
\end{abstract}

\section{INTRODUCTION}

The experimental implementation of quantum information processing (QIP) relies on identifying, coherently manipulating, coupling and detecting elementary excitations of individual quantum systems. All these operations need to be performed on a time scale much shorter than the decoherence time of the quantum system. This extremely challenging task has attracted the interest of an increasing number of experimentalists in all areas of science. Implementations of quantum logic operations are currently explored in a wide range of different quantum systems (1), e.g., nuclear magnetic spins in liquids and solids $(2 ; 3)$, ions in traps $(4 ; 5 ; 6 ; 7)$, atoms in microwave resonators (8), optical lattices (9), photonic band gap materials (10), Josephson junctions $(11 ; 12)$ or photons in quantum-optical systems $(13 ; 14)$. The complexity of this endeavor is quite clearly demonstrated by the fact that despite the outstanding progress in this field over the last few years, the most complex quantum calculation performed to date is the factorization of the number 15 (3).

A particularly attractive approach for realizing all-solid-state quantum information processing relies on using charge or spin excitations of semiconductor quantum dots (QD) as quantum bits. In QDs, electron and hole wave functions are localized in all three spatial dimensions on a nanometer length scale due to growth-induced nanoscale variations of the semiconductor composition. This makes QD interesting model systems for exploring the basic physics of quasi-zero-dimensional quantum confinement as well as interesting for building novel optical devices, such as low-threshold semiconductor lasers.

The optical and electronic properties of semiconductor QDs have been intensely studied during the last decade. Due to the pronounced and so far unavoidable growth-induced inhomogeneous broadening in ensembles of semiconductor QDs, the recent development of single QD spectroscopy has provided a wealth of new information (15). It is now understood that sufficiently confined QDs resemble in many respect atomic systems, showing atomic-like densities of states $(16 ; 17 ; 18)$, a shell-like absorption spectrum $(19)$ and - at low temperatures -

Further author information: (Send correspondence to C. Lienau)

C. Lienau: E-mail: lienau@mbi-berlin.de, Telephone: +49-30-6392-1476

T. Unold: present address: Hahn-Meitner-Institut Berlin, Glienicker Str. 100, D-14019 Berlin, Germany 
comparatively long dephasing times of up to $1 \mathrm{~ns}(20 ; 21)$. In addition, the large extent of the electron wave function in QDs gives rise to excitonic dipole moments of $10-100$ Debye, much larger than those of atomic systems. This strong coupling to light makes charge excitations of single quantum bits interesting for quantum information processing. Ultrafast light pulses with pulse durations in the $100 \mathrm{fs}$ range allow one to generate and manipulate exciton excitations of single QDs on a sub-picosecond time scale. With such ultrafast coherent carrier control, dephasing times in the 100 ps to 1 ns range can be considered as long, allowing in principal up to 10,000 coherent manipulations before decoherence destroys the quantum information stored in excitonic quantum bits (21). Another important consequence of the large excitonic dipole moments are comparatively strong dipolar interactions between adjacent quantum dots $(22 ; 23)$. Those interactions give rise to a nonlocal coupling between adjacent excitonic quantum bits, an important prerequisite for implementing scalable quantum gates. Consequently, different ideas for realizations of such gates have been proposed theoretically during the last years $(22 ; 23 ; 24 ; 25)$.

Such perspectives have triggered a research effort towards coherent control of excitonic excitations in semiconductor quantum dots. First successful experiments have shown coherent control on excited state transitions in the weak excitation regime $(26 ; 27 ; 28)$ before Rabi oscillations could be demonstrated on different quantum dot systems $(29 ; 30 ; 31 ; 32 ; 33)$. All these experiments have so far revealed a finite damping of Rabi oscillations, which has been attributed either to excitation-induced dephasing due to Coulomb interactions among charge excitations $(29 ; 34)$ or to exciton-phonon coupling $(35 ; 36)$. Most recently, an all-optical two-bit quantum logic gate has been demonstrated using the exciton and biexciton transitions of a single quantum dot (37).

In this paper, we present our recent experimental work on coherent control of excitonic excitations in quantum dots. We discuss a novel nano-optical technique (34) for probing optical nonlinearities of single quantum dots on ultrafast time scales $(34 ; 38)$. Coherent control of both amplitude (39) and phase (40) of the coherent exciton polarization in a single interface quantum dot is demonstrated and interactions between permanent excitonic dipole moments in a pair of neighboring quantum dots are resolved by analyzing Rabi oscillations in their nonlinear optical response (39).

The manuscript is structured as follows: In Sec. 2 we summarize the most important properties of the investigated samples. In Sec. 3 the experimental techniques are described. Results on coherent control of single quantum dots are given and discussed in Sec. 4 . In Sec. 5 we present first results on dipolar couplings between two quantum dots. Some conclusions are given sin Sec. 6 .

\section{INTERFACE QUANTUM DOTS}

An important QD model system are thin semiconductor quantum wells (QW). In quantum wells, local monolayer height fluctuations at the interfaces (interface roughness) and fluctuations of the alloy composition (alloy disorder) are unavoidable (Fig. 1(a)). The resulting disordered potential leads to the localization of excitons in single "interface" quantum dots with a confinement energy of about $10 \mathrm{meV}$ (Fig. 1(b)). This disorder gives rise to a pronounced inhomogeneous broadening of far-field optical spectra. In experiments with high spatial and spectral resolution, however, the smooth, inhomogeneously broadened photoluminescence (PL) spectra break up into narrow emission spikes from a few localized excitons $(16 ; 17 ; 18 ; 41 ; 42 ; 43)$.

The linear optical properties of interface QDs resemble in many aspects those of atomic systems. At low temperatures, the excitonic lines display a narrow homogeneous linewidth of $30-50 \mu \mathrm{eV}$, in agreement with measured dephasing times of 20-30 ps. The QDs show a discrete absorption spectrum (18) and often a fine structure splitting due to the spatial asymmetry of the monolayer islands. The temperature dependence of the exciton linewidth and the fine structure splitting has been thoroughly investigated $(18 ; 44)$. The correlation length of the disordered potential and thus also the center-of-mass wave function of localized excitons in interface quantum dots typically extends over several tens of nm, as known from near-field autocorrelation spectroscopy (42). This large coherence length of the excitonic wavefunction results in large QD dipole moments of 50100 Debye and a particularly strong coupling of these excitons to light $(45 ; 46)$. This makes interface quantum dots a particularly interesting model system for nonlinear spectroscopy of single quantum dots.

In this work, we investigate a sample consisting of 12 single QW layers of different thicknesses grown on a (100) GaAs substrate. The QW layers are separated by AlAs/GaAs short period superlattice barriers, each 
(a)
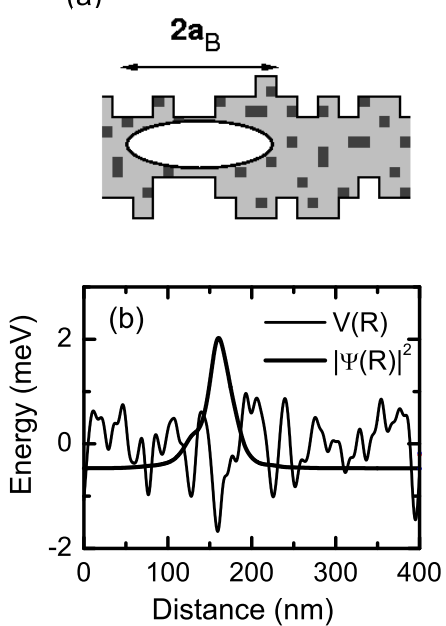
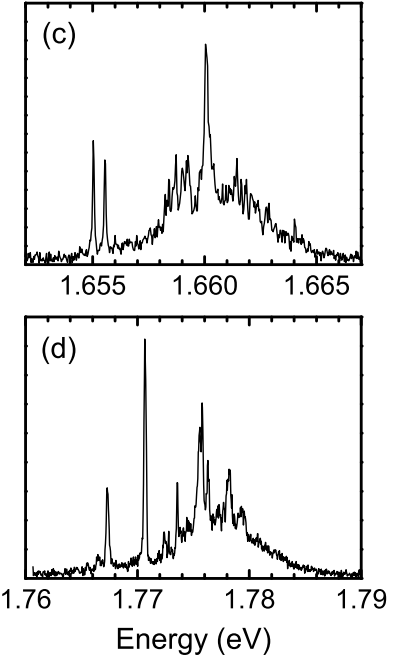

Figure 1. (a) Disorder in quantum wells arises from spatial fluctuations of the local quantum well thickness (interface roughness) and of the quantum well composition (alloy disorder). (b) Schematic illustration of the effective disorder potential $V(\mathbf{R})$ and of a localized excitonic center-of-mass wave function $|\Psi(\mathbf{R})|^{2}$. (c,d) Representative near-field PL spectra $(T=12 \mathrm{~K})$ of (c) a $5.1 \mathrm{~nm}$ thick and (d) a $3.3 \mathrm{~nm}$ thick (100) GaAs QW.

formed by nine AlAs and GaAs layers with a total thickness of $23.8 \mathrm{~nm}$. Here, we study the top seven QWs with thicknesses of 3.3 to $7.1 \mathrm{~nm}$. The layers are buried at distances between 40 and $211 \mathrm{~nm}$ below the surface. Growth interruptions of $10 \mathrm{~s}$ at each interface lead to a large correlation length of the QW disorder potential and to the formation of interface quantum dots (QD). The growth interruptions are kept short in order to avoid a monolayer splitting of the macroscopic PL spectra and to minimize the incorporation of impurities at the interfaces.

In Fig. 1(c) and (d) representative low temperature $(T=12 \mathrm{~K})$ near-field PL spectra are shown for the 3.3 and $5.1 \mathrm{~nm}$ thick (100) GaAs QW. The spectra reveal clearly the emission from excitons localized in interface quantum dots. The linewidth of the sharp resonances is limited by the spectral resolution of $100 \mu \mathrm{eV}$. The spectra are recorded at an excitation intensity of $110 \mathrm{nW}$, corresponding to an average excitation density well below one exciton per monolayer island. For excitation powers between 1 and $500 \mathrm{nW}$, we find a linear intensity dependence and an excitation-independent shape of the emission spectra, indicating negligible contributions from biexcitons and charged excitons.

In addition to the sharp localized exciton emission, these spectra display at higher energies a spectrally broad background emission from more delocalized excitons in QW continuum states (47). This is a disadvantage for QIP applications, as it may be difficult to avoid the uncontrolled population of such delocalized exciton states when ultrashort and thus spectrally broadband pulses are used for optical excitation. Yet, we will demonstrate below that such problems can be reduced by careful spectral shaping of the excitation pulses. Important properties of IQD are the excellent interface quality of the (100) GaAs quantum wells and the strong reduction of piezoelectric and strain fields. In the investigated samples, the energetic positions of the sharp exciton emission lines remains unchanged over many hours and we observe no signs of a spectral diffusion of the exciton lines.

\section{COHERENT SPECTROSCOPY ON INTERFACE QUANTUM DOTS: EXPERIMENT}

In the experiments on interface quantum dots (IQD), we read-out quantum information from single interface quantum dots by directly probing the transient nonlinear optical spectrum of ground-state exciton transitions of a single quantum dot with sub-picosecond time resolution. Our experimental concept is outlined in Fig. 2(a). We use spectrally broad femtosecond laser pulses, centered around the excitonic QW absorption resonance, coupled into a near-field fiber probe to probe the optical QD nonlinearity. As a near-field probe we use an uncoated 
etched single mode optical fiber taper with a cone angle of about $30^{\circ}(48)$. With such probes we reach - in an illumination/collection geometry - a spatial resolution of about $150 \mathrm{~nm}$, i.e., about $\lambda / 5$ (41). This high spatial resolution together with their large collection efficiency makes such uncoated fiber probes particularly well suited for semiconductor nano-spectroscopy. Experimentally, we find that for GaAs samples about $1 \%$ of the light coupled into the fiber is collected in this illumination/collection geometry.

(a)

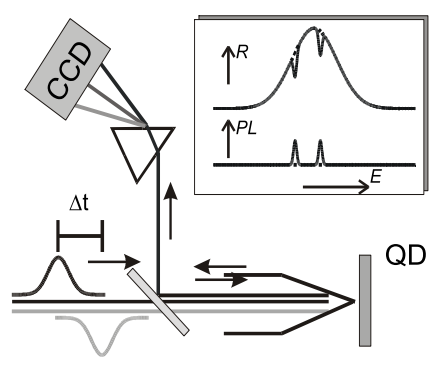

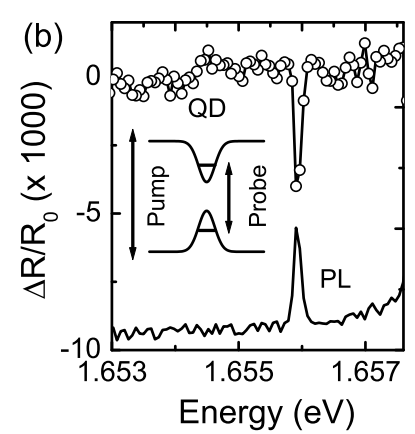

Figure 2. (a) Schematic illustration of the experimental setup and of near-field PL and reflectivity spectra of the QD sample. (b) Near-field PL spectrum of a single QD (solid line) and differential reflectivity spectrum $\Delta R / R_{0}$ at $\Delta t=30 \mathrm{ps}$. $\mathrm{PL}$ and $\Delta R$ are recorded with identical pump pulses centered at $1.675 \mathrm{eV}$, exciting electron-hole pairs in $2 \mathrm{D}$ continuum states. The $100 \mathrm{nW}$ probe pulses of $19 \mathrm{meV}$ bandwidth are centered at $1.655 \mathrm{eV}$, around the QD absorption resonance. Inset: Schematic energy diagram.

In the pump-probe experiments, the probe laser light reflected from the QW sample is collected by the same fiber probe, dispersed in an $0.5 \mathrm{~m}$ monochromator and then detected with a high sensitivity liquid-nitrogen cooled CCD camera. This steady-state reflectivity spectrum $R_{0}\left(\omega_{\text {det }}\right)$ contains weak spectrally narrow resonances from single QD transitions (Fig. 2(a)).

The interaction with a second pump pulse now affects the QD spectrum and thus gives rise to a modified probe reflectivity $R\left(\omega_{\text {det }}\right)$. Differential probe reflectivity spectra $\Delta R\left(\omega_{\text {det }}, \Delta t\right) / R_{0}=\left[R\left(\omega_{\text {det }}, \Delta t\right)-R_{0}\left(\omega_{\text {det }}\right)\right] / R_{0}\left(\omega_{\text {det }}\right)$ are recorded at a fixed spatial position of the near-field tip as a function of the time delay $\Delta t$ between pump and probe pulses. To probe the nonlinear optical response from single quantum dots, the high spatial resolution of the near-field technique is needed for two reasons. First, the combined spatial and spectral resolution allows us to isolate single QD resonances (Fig. 1). Second, the relative amplitude of the QD resonance in $R_{0}\left(\omega_{\text {det }}\right)$ scales, in first approximation, inversely proportional to the square of the spatial resolution.

We assume for simplicity that the QD absorption spectrum can be modeled as that of an ideal two-level system (TLS) and that the incident laser power is homogeneously distributed over an area $A$ (the areal resolution of the microscope). Then the incident power is $P=I_{0} A$ and the absorbed power is $P_{Q D}=I_{0} \sigma_{Q D}$, with $I_{0}$ being the incident intensity and $\sigma_{Q D}$ the QD absorption cross section. For an ideal TLS,

$$
\sigma_{Q D}(\omega)=\frac{\omega \mu_{Q D}^{2}}{n c \epsilon_{0} \hbar} \frac{\gamma}{\gamma^{2}+\left(\omega-\omega_{Q D}^{2}\right)},
$$

where $\omega_{Q D}=2 \pi c / \lambda_{Q D}$ denotes $\mathrm{QD}$ resonance frequency, $\omega$ the laser frequency, $\mu_{Q D}$ the QD dipole moment, $n$ the refractive index and $\gamma=1 / T_{2}$ the dephasing rate of the QD polarization. Thus improving the resolution from $1 \mu \mathrm{m}$ to $100 \mathrm{~nm}$ increases the weak nonlinear QD signal by two orders of magnitude. For values typical for our experiments, $A=(250 \mathrm{~nm})^{2}, \mu_{Q D}=60 \mathrm{D}, \gamma=\left(30 \mathrm{ps}^{-1}\right), n=3.5, \lambda_{Q D}=750 \mathrm{~nm}$, we estimate $\sigma_{Q D} / A \simeq 0.04$, well measurable with the sensitivity of our setup.

The near-field PL and differential reflectivity $\Delta R$ spectrum of a single QD are compared in Fig. 2(b). To record the $\Delta R$ spectrum we use $100 \mathrm{fs}$ pump pulses derived from a $80 \mathrm{MHz}$ repetition rate Ti:sapphire laser. The pump pulses in this experiment create less than five electron-hole pairs in QW states, corresponding to an excitation density of $5 \cdot 10^{9} \mathrm{~cm}^{-2}$. Relaxation of these extra carriers into the QD bleaches the QD absorption and this bleaching is probed with $1 \mathrm{fJ}$ probe pulses centered around the QW absorption resonance. Fig. 2(b) depicts 
a differential reflectivity spectrum $\Delta R\left(E_{\text {det }}\right)$ at a time delay of $30 \mathrm{ps}$ in the low energy region of the $5.1 \mathrm{~nm} \mathrm{QW}$ absorption spectrum. It displays a single spectrally sharp resonance at exactly the same spectral position $E_{Q D}$ as the simultaneously recorded near-field PL spectrum. The large amplitude of the signal of $5 \cdot 10^{-3}$ is consistent with a spatial resolution of the experiment of 200-250 nm. Two-dimensional spatial scans indicate a resolution of $230 \mathrm{~nm}$, partly limited by the QW-to-surface distance.

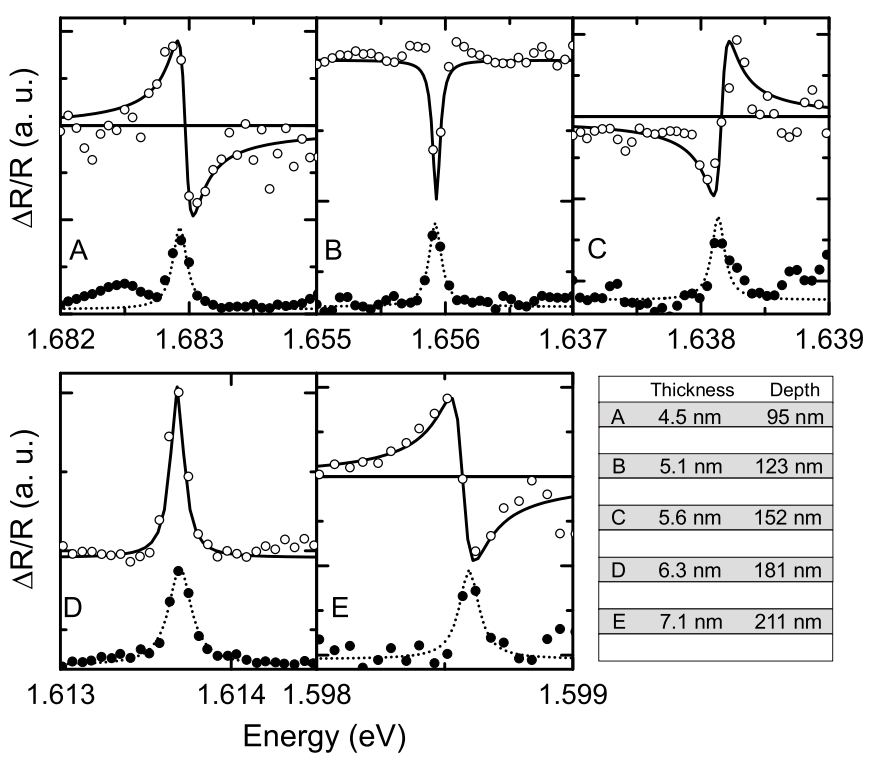

Figure 3. Differential reflectivity spectra (open circles) of five interface QDs located at different depths of 95 to $210 \mathrm{~nm}$ below the sample surface (see inset). The differential reflectivity spectra are compared to simultaneously recorded PL spectra. Note the transition between dispersive and absorptive line shapes.

To better understand the image contrast in these pump-probe experiments, we compare in Fig. 3 differential reflectivity $\Delta R\left(E_{\text {det }}\right)$ and PL spectra recorded under similar excitation conditions for single localized excitons in five different QWs buried at distances of $95 \mathrm{~nm}$ to $211 \mathrm{~nm}$ below the surface. We very clearly observe a transition between a dispersion-like and an absorption-like line shape as the QW-to-surface distance is varied. This behavior of the QD line shape can be understood in the framework of a local oscillator model as caused by the interference between the electric probe laser field $E_{R}(t)$ reflected from the sample surface and the field $E_{Q D}(t)$ emitted from the QD in back direction. Our experiment works in the following way (Fig. 4). A fraction $E_{R}(t)$ of the probe laser transmitted through the near-field probe is reflected from the sample surface and coupled back into the near field fiber probe. The probe field $E_{T}(t)$, transmitted into the semiconductor, induces a polarization $P_{Q D}(t)=\int d t^{\prime} \chi_{Q D}\left(t^{\prime}\right) E_{T}\left(t-t^{\prime}\right)$ of the QD located at a distance $d$ below the sample surface. Here, $E_{T}(t)$ and $\chi_{Q D}$ denote the probe field interacting with the QD and the QD susceptibility, respectively. The QD polarization re-emits an electric field and a fraction of this field, $E_{Q D}(t)$ is locally collected by the near-field probe where it interferes with $E_{R}(t)$.

The time-integrated reflectivity $R(\omega)$ detected behind the monochromator is proportional to $\mid \tilde{E}_{Q D}(\omega)+$ $\left.\tilde{E}_{R}(\omega)\right|^{2} \simeq\left|\tilde{E}_{R}(\omega)\right|^{2}+2 \operatorname{Re}\left[\tilde{E}_{R}^{*}(\omega) \tilde{E}_{Q D}(\omega)\right]$, where $\tilde{E}(\omega)$ denotes the Fourier transform of the field $E(t)$. Here, the finite monochromator resolution and the weak contribution from $\left|\tilde{E}_{Q D}\right|^{2}$ has been neglected. Excitation by the pump laser affects the QD polarization and thus results in a change of the QD reflectivity. The differential reflectivity $\Delta R(\omega, \Delta t)$ represents the spectral interferogram of $\tilde{E}_{R}$ and $\tilde{E}_{Q D}$ :

$$
\Delta R(\omega, \Delta t) \propto \operatorname{Re}\left\{\tilde{E}_{R}^{*}(\omega)\left[\tilde{E}_{Q D}(\omega, \Delta t)-\tilde{E}_{Q D, 0}(\omega)\right]\right\} .
$$

The spectral shape of this interferogram evidently depends on the QD polarization dynamics and on the phase delay between $E_{Q D}(t)$ and $E_{R}(t)$. Treating the QD for simplicity as a point dipole and the near-field tip as a point-like emitter, the phase delay and thus the spectral shape of this interferogram depends on the distance 


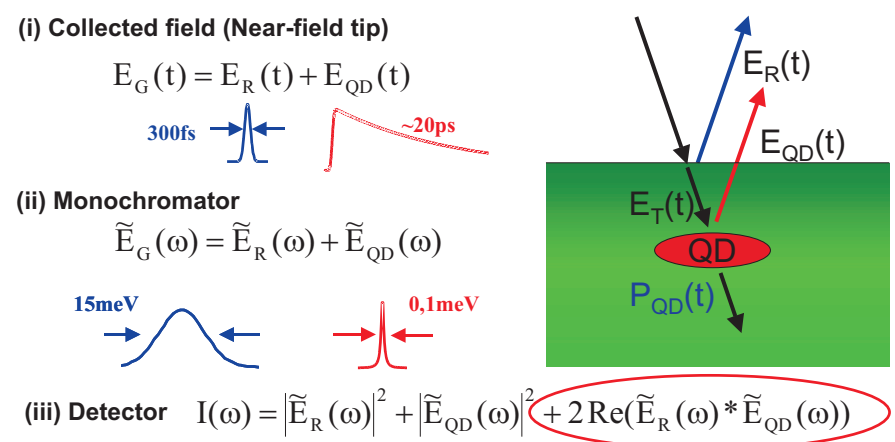

Figure 4. Heterodyne detection of coherent QD polarizations. A fs probe laser is coupled through a near-field fiber probe. A large fraction $E_{R}$ of the probe laser is directly reflected the sample surface into the fiber probe. The transmitted probe light $E_{T}$ is induces a QD dot polarization $P_{Q D}$ and the fraction $E_{Q D}$ of the electric field re-emitted from the QD is collected by the near-field probe. $E_{R}$ and $E_{Q D}$ are spectrally dispersed in a monochromator and interfere on the CCD detector. This heterodyne detection scheme greatly enhances the weak QD field.

between quantum dot and near field tip. This interference effect is nicely seen in Fig. 3 and explains the transition between absorptive and dispersive line shapes. Since the QDs are buried more than $50 \mathrm{~nm}$ below the surface, the near-field terms of the QD dipole emission can be neglected since they decay on a typical length scale of $\lambda /(2 \pi n) \simeq 35 \mathrm{~nm}(n \simeq 3.5$ - refractive index). Based on an optical path of $4 \pi n d / \lambda$, we estimate a phase change of $\pi / 2$ for a change in QD-sample distance of $28 \mathrm{~nm}$. This is in quite good agreement with the results of Fig. 3 . We consider this convincing evidence for the validity of the phenomenological local oscillator model described above. Clearly a detailed analysis of these data, using, e.g., a Green function solution of Maxwell's equations for a realistic experimental geometry is desirable for a quantitative comparison between experiment and theory.

\section{COHERENT CONTROL IN SINGLE INTERFACE QUANTUM DOTS}

In this section, we describe experiments probing the coherent polarization dynamics of a single interface quantum dot induced by impulsive excitation with ultrafast light pulses. Specifically, three different topics are addressed. First, we ask the fundamental question to what extent the ultrafast nonlinear optical response of a single IQD resembles that of an atomic system and how many-body Coulomb interactions - often governing optical nonlinearities of higher-dimensional systems such as quantum wells and wires - affect the QD polarization dynamics. Then we demonstrate coherent control of the phase of the QD polarization by probing the optical Stark effect in a single QD and coherent control of the polarization amplitude by probing Rabi oscillations in single QD.

\subsection{Ultrafast optical nonlinearities of single interface quantum dots}

To study the effects of many-body interactions on the QD nonlinearities, we perform a quasi-two-color pumpprobe experiment, exciting the QD sample in the QW absorption continuum with $100 \mathrm{fs}$ pump-pulses with a pulse energy of $1.5 \mathrm{fJ}$. These pulses create carriers in QW states and the resulting change in the QD spectrum is probed. The dynamics of the spectrally resolved reflectivity change measured on different QD resonances is shown in Fig. 5. After a picosecond rise of the signal at negative delay times (probe precedes pump) one finds a partial decay with a time constant of about $6 \mathrm{ps}$, followed by a much slower decay with time constants of 50 to $150 \mathrm{ps}$, depending on the specific QD resonance investigated.

The nonlinearities observed at sufficiently long positive $\Delta t$ are easily understood on the basis of a simple twolevel model for the QD nonlinearity. The pump laser creates a non-equilibrium distribution of electron-hole pairs in QW continuum states. Subsequent trapping of these carriers gives rise to a bleaching of the QD absorption and a concomitant decrease of the QD absorption. Hence, the decay time reflects the lifetime of the individual exciton state probed. Following an earlier conjecture (18), the QD population decay is mainly dominated by radiative recombination, i.e. $\tau_{r a d} \simeq \tau_{Q D}$. We can then estimate the dipole moment of the individual QDs using $(49 ; 46)$ :

$$
\frac{1}{\tau_{\text {rad }}}=n \frac{\omega^{3} \cdot \mu_{Q D}^{2}}{3 \pi \epsilon_{0} \hbar c^{3}}
$$



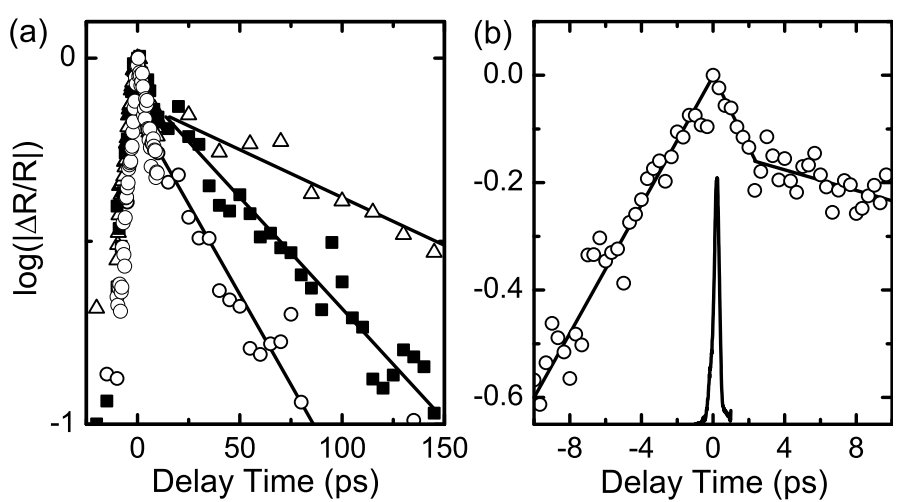

Figure 5. (a) Temporal dynamics of $\Delta R / R$ for three different QD resonances (logarithmic ordinate scale). All decays are biexponential with a slow decay time varying between 30 and 150 ps. (b) Early time $\Delta R / R_{0}$ dynamics of a single QD resonance. A slow rise of $\Delta R / R_{0}$ is observed at negative time delays. The time resolution of the experiment is $150 \mathrm{fs}$, as indicated by the cross-correlation measurement (solid line around $\Delta t=0$ ).

We estimate dipole moments $\mu_{Q D}$ of 50 to 85 Debye for $\tau_{\text {rad }}$ between 150 and 50 ps. These values are in rather good agreement with previous estimates $(29 ; 49)$. They exceed those of atomic systems by more than an order of magnitude and reflect the large spatial extension of the exciton center-of-mass wave function in these QDs. Near-field autocorrelation spectra indicate an exciton localization length of about 40-50 $\mathrm{nm}$ (50). Due to the statistical nature of the disorder potential, the exciton localization length and thus the dipole moment and radiative recombination rate varies quite strongly from QD to QD, as seen in Fig. 5(b). Theoretical models of localized excitons in disordered quantum wells $(51 ; 50)$ yield comparable results.
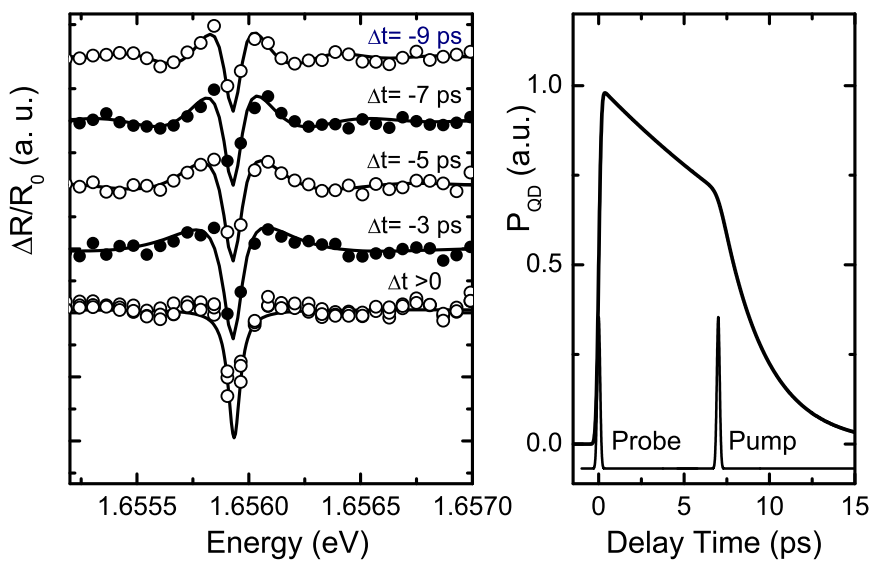

Figure 6. (a) Near-field $\Delta R / R_{0}$ spectra (circles) at different delay times $\Delta t$. The spectra at $\Delta t<0$ display pronounced spectral oscillations around the excitonic resonance. The solid lines shows simulated spectra for the perturbed free induction decay of the coherent QD polarization assuming $T_{2}=15$ ps. (b) Dynamics of $P_{Q D}(t)$ extracted from the timedependent near-field $\Delta R / R_{0}$ spectra.

The dynamics of the QD reflectivity on a time scale of less than $10 \mathrm{ps}$, however, are quite different from what is expected for an ideal atomic system. The time evolution shows an 8-ps rise at negative delay times, much slower than the 150 -fs cross correlation of pump and probe pulses. A biexponential decay is found at positive delays, where the slow component reflects the exciton lifetime as discussed above. The fast decay time of about 6 ps is similar for all different QD's. The spectral characteristics of the differential reflectivity are markedly different at positive and negative delays. At negative delays, pronounced spectrally symmetric oscillations around the excitonic resonance are observed Fig. 6. Their oscillation period decreases with increasing negative time delay. At large positive delays, the spectra show a bleaching of the QD resonance (34). 
This complex behavior reflects directly the coherent polarization dynamics of the excitonic QD excitation. To account for this behavior, one has to consistently describe the dynamics of the field $E_{Q D}(t)$ radiated from the coherent QD polarization $P_{Q D}(t)$. We phenomenologically describe the QD as an effective two-level system with a ground, no-exciton state $|0\rangle$, and an excited one-exciton state $|1\rangle$. Then the quantum state of the TLS is given as a coherent superposition $|\psi(t)\rangle=c_{0}(t)|0\rangle+c_{1}(t)|1\rangle$. Within the density matrix formalism, $P_{Q D}(t)$ is given as $P_{Q D}(t)=\mu_{Q D}^{*} \rho_{01}+$ c.c., where the microscopic QD polarization $\rho_{01}=\left\langle c_{0}^{*} c_{1}\right\rangle, \mu_{Q D}$ denotes the QD dipole moment and $\langle\ldots\rangle$ the ensemble average (52). Then, the well known Bloch equations hold and $\rho_{01}$ obeys the equation of motion

$$
\frac{\partial}{\partial t} \rho_{01}(t)=-i \omega_{Q D} \rho_{01}(t)+i\left(1-2 n_{Q D}\right) \omega_{R}-\gamma \rho_{01}(t),
$$

with exciton energy $\omega_{Q D}$, dephasing rate $\gamma$, exciton population $n_{Q D}$ and generalized Rabi frequency $\omega_{R}$.

In the absence of a pump laser, the resonant probe laser impulsively excites a coherent QD polarization which then decays with the dephasing rate $\gamma$ (free induction decay). The re-emitted field interferes with the reflected probe laser field, giving rise to a Lorentzian QD line shape in $R_{0}(\omega)$. The fact that we observe a linewidth that is limited by our monochromator resolution of about $60 \mu \mathrm{eV}$ gives a lower limit for the QD dephasing time of $T_{2}=1 / \gamma>15 \mathrm{ps}$.

The transient spectral oscillations around the QD exciton resonance at negative time delays indicate that this free induction decay of the $P_{Q D}(t)$ is perturbed by the presence of the pump laser. In semiconductors, such oscillations have so far only been observed for higher dimensional system, e.g., studies of transient QW nonlinearities $(53 ; 54)$. In our experiments, the off-resonant pump does not directly interact with the QD dipole but creates electron-hole pairs (density $n_{Q W}$ ) in the QW continuum. Thus many-body interactions perturb the free induction decay of $P_{Q D}(t)$.

The spectra at $\Delta t<0$ are quantitatively described by assuming that an excitation-induced dephasing (55), i.e., an increase in $\gamma$ due to the interaction between $\rho_{01}$ and $n_{Q W}$ is the leading contribution to the QD nonlinearity at early times. Coulomb scattering between the QD dipole and the initial nonequilibrium carrier distribution in the QW causes this additional fast damping of $\rho_{01}$. In the frequency domain, this excitation-induced dephasing leads to oscillatory structures in the spectrum with a period determined by the time delay between probe and pump. The solid lines in Fig. 6(a) are calculated from Eq. (4) by assuming that the probe-induced QD polarization $P_{Q D}(t)$ decays initially with an effective dephasing time $T_{2}=15 \mathrm{ps}$, decreasing to $T_{E I D}=3 \mathrm{ps}$ after the arrival of the pump laser (Fig. 6(b)). Such an excitation-induced dephasing model accounts quantitatively for the the transient oscillations and this analysis allows to extract the QD polarization dynamics. A detailed theoretical analysis of the data was performed on the basis of the semiconductor Bloch equations in the mean-field approximation (34) gives strong for this interpretation.

The assumption of a density-dependent dephasing rate can also explain the fast decay of the differential reflectivity at early positive delay times (Fig. 5(b)). We have to assume that $n_{Q W}$ decays on a time scale of about 3 ps. This decay is most likely due to carrier trapping into QD states. Then, the initial fast differential reflectivity decay reflects the transition from a QD nonlinearity dominated by excitation-induced dephasing to a nonlinearity dominated by exciton bleaching due to the population relaxation into the QD.

These results highlight two important features of interface quantum dots. First, the coherence of the excitonic QD polarization persists for more than 10 ps even after resonant excitation with spectrally broad-band femtosecond pulses. This decoherence times is two orders of magnitude larger than the duration of the excitation pulses. On the other hand Coulomb many-body interactions may contribute significantly to their optical nonlinearities on ultrashort time scales if an additional exciton population in quantum well continuum states is created during the optical excitation process. Such many-body interactions have to be taken into account as important additional dephasing mechanisms.

\subsection{Optical Stark effect and Rabi oscillations in a quantum dot: Ultrafast control of single exciton polarizations}

In this subsection, we demonstrate coherent control of both the amplitude and phase of the coherent QD polarization on an ultrafast time scale. Coherent control of the population of a generic two-level system with finite 
electronic dipole moment $\vec{\mu}$ can be achieved by resonant impulsive excitation with light pulses much shorter in duration than the decoherence time of the microscopic polarization $\rho_{01}$. Neglecting for simplicity the finite decoherence time of this TLS, the excited state population after the interaction with the excitation laser is given as $n_{1}=\sin ^{2}(\theta / 2)$, with $\theta$ being the pulse area

$$
\theta=\frac{\vec{\mu} \cdot \hat{\varepsilon}}{\hbar} \int_{-\infty}^{\infty} E(t) d t,
$$

where $E(t)$ denotes the time-dependent electric field profile of the excitation laser and $\hat{\varepsilon}$ its polarization direction. Thus, for weak excitation pulses the excited state population first increases linearly with increasing pulse intensity until it reaches a value of $n=1$ for $\theta=\pi$, i.e., until the two-level-system is fully inverted. Further increase in the pulse intensity induces stimulated emission from the excited state back to ground state and thus a decrease of excited state population. After interaction with a light pulse of area $\theta=2 \pi$ the excited state population reaches again $n=0$, i.e. the system is back in its original state. For higher excitation, the population shows the well known Rabi oscillations. Of course this simplified picture only holds for resonant excitation and negligible decoherence and thus the study of Rabi oscillations should generally give insight into the decoherence of quantum systems in the strong excitation regime.

(a)

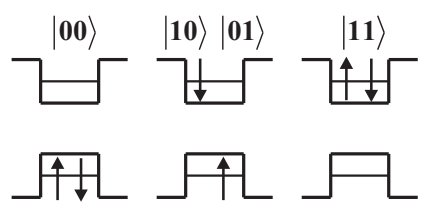

(c)

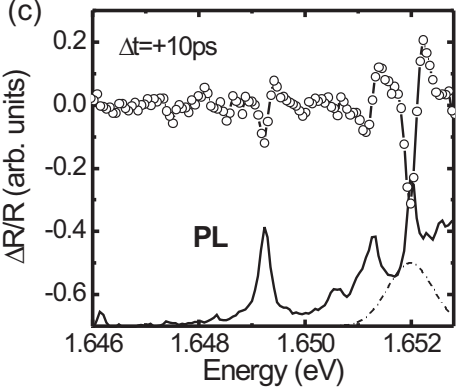

(b)

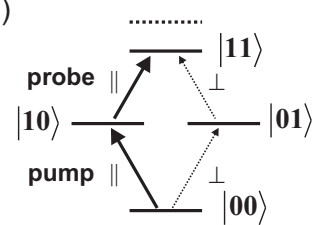

(d)

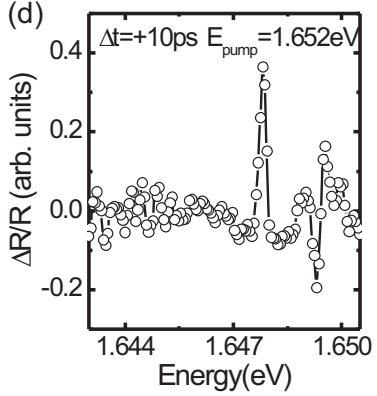

Figure 7. (a) Schematics of exciton ground $(|00\rangle)$, one-exciton $(|10\rangle$, and $|01\rangle)$, and biexciton states $|11\rangle$ in a QD. (b) Excitation-level diagram in an interface QD and optical selection rules for pump and probe laser. (c) PL and $\Delta R$ for above band gap excitation. In the studies of biexciton nonlinearities, the pump laser (dashed line) is tuned to the exciton resonance at $1.652 \mathrm{eV}$. (d) Pump-induced biexciton nonlinearity. The time delay between pump (at $1.652 \mathrm{eV}$ ) and probe laser is $\Delta t=10 \mathrm{ps}$.

For resonant excitation of only a single excitonic transition, we use spectrally tailored optical pulses with a spectral width of about $1 \mathrm{meV}$ and a pulse duration of about $1.5 \mathrm{ps}$. We tune these pump pulses to a specific quantum dot resonance and probe the induced optical nonlinearity with collinearly polarized 15-meV broad of $200 \mathrm{fs}$ duration. The idea of the experiment is to read-out the exciton population after the QD interaction with the pump pulse by probing the induced absorption on the exciton - biexciton transition. Since each confined electron state in the QD can be occupied with two electrons of opposite spin orientation, two distinguishable single exciton states with orthogonal polarization can be optically excited. Simultaneous excitation of both exciton states results in a transient population of the bound biexciton state [Fig. 7(a)]. In IQDs, the biexciton energy is normally slightly smaller (1-4 meV) than the sum of the two exciton energies due to the Coulombic interaction between the two excitons. Since the monolayer islands in interface quantum dots are slightly elongated along the [-110] direction (18), the energetic degeneracy of the two single-exciton states is lifted (by typically less than 100 $\mu \mathrm{eV})(18)$ and one finds linear polarization selection rules for the exciton and biexciton transitions (18; 56). The energy level structure of the QD states and the polarization selection rules can be summarized in a four-level 
system for the no-exciton crystal ground state $|00\rangle$, two exciton states with orthogonal polarization $|10\rangle$ and $|01\rangle$ and the biexciton state $|11\rangle$ [Fig. 7(b)]. Optical excitation of the $|00\rangle \rightarrow|10\rangle$ single exciton transition gives rise to excited state absorption on the $|10\rangle \rightarrow|11\rangle$ transition. This excited state absorption is not present in the absence of single exciton excitation. Experimentally we observe indeed, after single exciton excitation at $1.652 \mathrm{eV}$ [Fig. 7(c)] a new transition resonance red shifted by $1.648 \mathrm{eV}$, red-shifted by $4 \mathrm{meV}$ from the single exciton transition which is assigned to the exciton-biexciton $X \rightarrow X X$ transition [Fig. 7(d)]. Also the dynamics of the pump-probe signal on the $X \rightarrow X X$ is consistent with this assignment [Fig. 8(a)] . We observe no biexciton nonlinearity at negative delay times (probe precedes pump), i.e. in the absence of $|10\rangle$ exciton excitation. Around time zero the $X \rightarrow X X$ signal rises within the time resolution of the experiment and then decays exponentially on a $40 \mathrm{ps}$ time scale, i.e., with the radiative single exciton lifetime. This means that the amplitude of the induced $X \rightarrow X X$ reflectivity change is a direct measure of the transient $|10\rangle$ exciton population generated by the pump pulse. We study the effect of the pump power on this biexciton nonlinearity. The experimental results at a delay time of $\Delta t=10 \mathrm{ps}$ are shown in Fig. 8(b). The magnitude of the differential biexciton nonlinearity $\Delta R_{X X}$ displays pronounced oscillations when varying the power of the pump laser $P_{p u}$. In Fig. 8(b) $\Delta R_{X X}$ is plotted vs. the maximum field strength $E_{p u} \propto \sqrt{P_{p u}}$ of the pump laser. These oscillations give clear evidence for Rabi oscillations on a single ground-state exciton transition in a single interface quantum dot. Despite the clarity of these oscillations, the experiment also shows that interface quantum dots are not an ideal two-level system. The biexcitonic nonlinearity at the second maximum, corresponding to a $3 \pi$ excitation pulse, is about $1 / 3$ smaller than that at the first maximum - corresponding to a $\pi$ excitation. This unwanted damping of the Rabi oscillations is caused again by excitation-induced dephasing as an additional source of decoherence. The field dependence of the biexciton nonlinearity, $\Delta R_{X X}\left(E_{p u}\right)$, can well be reproduced within the framework of Optical Bloch Equations of a two-level system with an intensity-dependent dephasing rate $\gamma=1 / T_{2}+\gamma_{1} \cdot E_{p u}^{2}$ [Fig. 8(b)]. Good agreement between experiment and model is achieved by assuming a dipole moment of $60 \mathrm{D}$, similar to those previously measured. The microscopic physics underlying this excitation-induced dephasing is similar to that reported for above band gap excitation of QW continuum states in Sec. 4.1. Excitation by the ps-pump pulse not only drives the desired single exciton transition but also creates coherent polarizations and incoherent populations in the QD environment. Since our pump-probe signals are accumulated over a large number of typically $10^{8}$ laser pulses, these unwanted excitations effectively give rise to a fluctuating QD environment and, thus, to decoherence of the ensemble-averaged QD polarization. Our experimental results are well reproduced by assuming that the QD dephasing rate increases from less than $(15 \mathrm{ps})^{-1}$ (an upper limit given by our finite monochromator resolution) to about $(6 \mathrm{ps})^{-1}$ for a pulse area of $3 \pi$. In summary, these experiments evidence coherent control of the population of a single QD exciton by demonstration of Rabi oscillations.
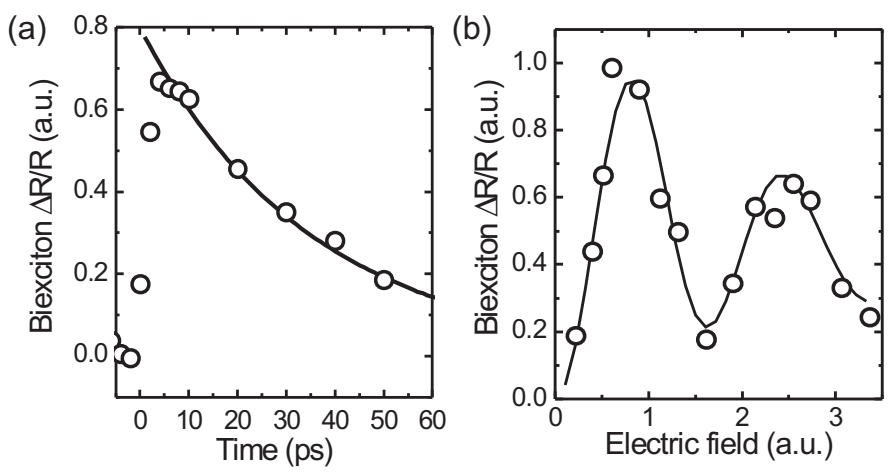

Figure 8. (a) Temporal dynamics of the biexciton nonlinearity at $1.648 \mathrm{eV}$. The excitation conditions are as shown in Fig. 7. The $40 \mathrm{ps}$ decay at $\Delta t>0$ reflects the exciton lifetime. (b) Rabi oscillation is single IQD. Magnitude of the biexcition nonlinearity as function field amplitude of the pump laser at $\Delta t=10 \mathrm{ps}$.

Full coherent control over the single exciton excitation, however, requires not only control over the exciton population or more precisely the amplitude of the microscopic polarization $\rho_{01}$ but also control over the polarization phase $\phi=\arctan \left(\operatorname{Im}\left(\rho_{01}\right) / \operatorname{Re}\left(\rho_{01}\right)\right)$. In a Bloch sphere representation, often used to visualize the quantum dynamics of quasi-two-level systems, the momentary polarization $\rho_{01}$ is represented as a three-dimensional vec- 
tor $\left.\rho_{B}=\left[\operatorname{Re}\left(\rho_{01}\right), \operatorname{Im}\left(\rho_{01}\right)\right), 1 / 2\left(n_{1}-n_{0}\right)\right]$, with $n_{i}=\left\langle c_{i}^{*} c_{i}\right\rangle$ describing the population of state $|i\rangle$. In this representation, polarization control thus means controlling the azimuthal angle $\phi$ in the $\left[\operatorname{Re}\left(\rho_{01}\right), \operatorname{Im}\left(\rho_{01}\right)\right]$-plane. Simultaneous control over amplitude and phase of the polarization thus gives full control over both degrees of freedom on the Bloch sphere.

Here we demonstrate control of the relative phase between the driving laser and the excitonic polarization by making use of the optical Stark effect (OSE). The OSE is one of the fundamental coherent light-matter interactions describing the light-induced shift ('dressing') of energy levels in the presence of non-resonant laser fields. In atomic systems the OSE is well known and, for weak excitation, well described by optical Bloch equations for independent two-levels systems $(57 ; 58)$. In higher-dimensional semiconductors, e.g. quantum wells, however, the polarization dynamics induced by non-resonant light fields is much more complex than in atomic systems and often dominated by Coulomb-mediated many-body interactions $(59 ; 60 ; 61 ; 62)$. Effects such as exciton-exciton interaction, biexciton formation or higher-order Coulomb correlations may affect the magnitude of the energy shift, the exciton oscillator strength and may even reverse the sign of the shift $(61 ; 63 ; 64 ; 65 ; 66)$. Here, we report the first experimental study of the OSE in a single quasi-zero-dimensional semiconductor quantum $\operatorname{dot}(40)$.
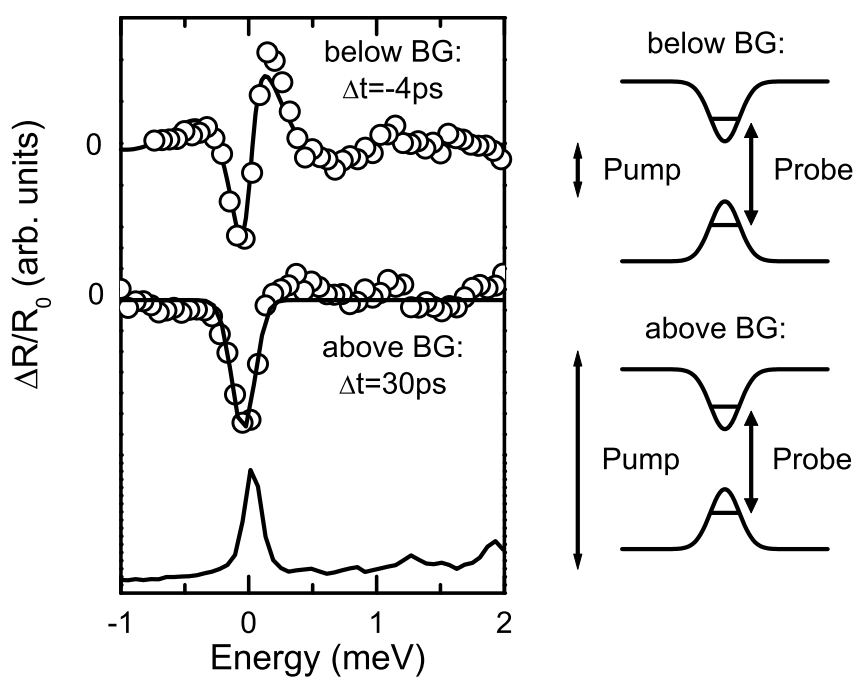

Figure 9. (left) PL spectrum of a single QD resonance $\omega_{Q D}=1.6503 \mathrm{eV}$ and differential reflectivity $\Delta R(\omega) / R_{0}$ for above band gap excitation at $\Delta t=30 \mathrm{ps}$ and for below band gap excitation at $\Delta t=-4$ ps with 2-ps pulses at $1.647 \mathrm{eV}$ (bandwidth $\sigma=0.8 \mathrm{meV})$ in the weak excitation limit $\left(P_{p u}=0.12 \mu \mathrm{W}\right)$. Solid lines: Bloch equation model. (right) Schematic excitation diagram.

Fig. 9 compares the PL (solid line) from a single QD and the $\Delta R(\omega, \Delta t=30 \mathrm{ps}) / R_{0}$ spectrum for above band gap excitation of QW continuum states (solid circles). The absorptive $\Delta R$ spectrum reflects the bleaching of the QD resonance as described above. For below band gap excitation, however, we observe for weak excitation $\left(P_{p u}<0.2 \mu \mathrm{W}\right)$ and negative delay times, here $\Delta t=-4$ ps (probe precedes pump) a dispersive line shape centered around $\omega_{Q D}$. With increasing excitation power, we find a drastic change in the line shape of $\Delta R(\omega)$ [Fig. 10(a)]: For strong excitation, the signal maximum shifts slightly towards higher energies and an increasing number of spectral oscillations is observed, in particular on the high energy side of the QD resonance. This change in line shape occurs together with a saturation of the strength of the nonlinear signal $\Delta R_{m}$, taken as the difference between minimum and maximum of $\Delta R(\omega)$ [Fig. 10(b)]. As we will show below, this characteristic change in line shape allows us to extract the phase shift $\Delta \phi$ of the QD polarization due to the interaction with the off-resonant pump laser from a Bloch equation simulation. The extracted phase shift $\Delta \phi$ is plotted as a function of excitation power in Fig. 10(c).

To ensure that we are indeed probing only a light-induced shift of the exciton resonance, we also plot the time evolution of the QD nonlinearity $\Delta R_{m}(\Delta t) / R_{0}$ [Fig. 11]. It is important that the signal vanishes completely for 
positive delay times $\Delta t>0$ (pump precedes probe) and rises around $\Delta t=0$ within the time resolution of our experiment of 250 fs. For $\Delta t<0, \Delta R_{m}(\Delta t)$ decays with a time constant of $\tau_{d}=8$ ps.
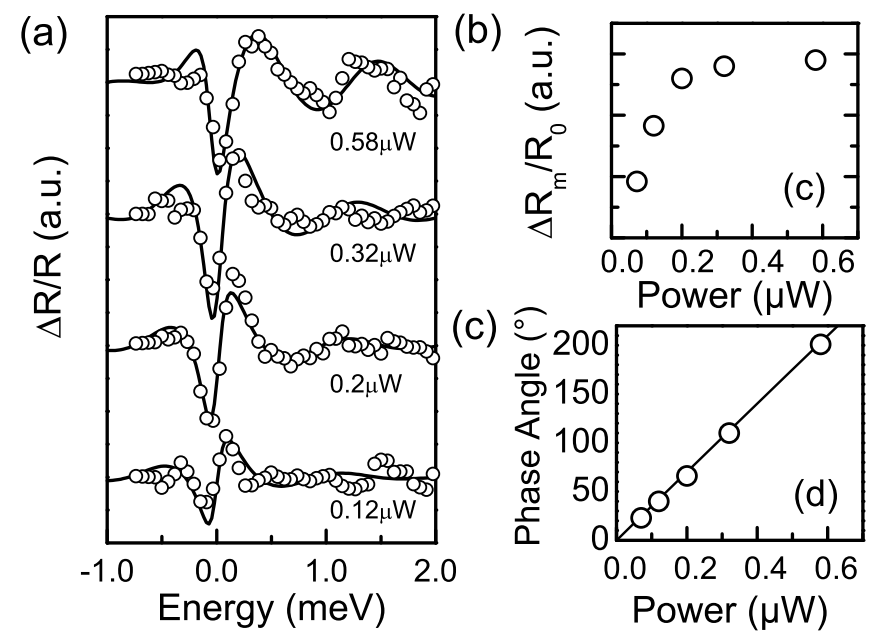

Figure 10. (a) Optical Stark Effect in a single QD. Differential reflectivity spectra $\Delta R(\omega) / R_{0}$ for below band gap excitation at $\Delta t=-4$ ps with 2-ps pulses at $1.647 \mathrm{eV}$ (bandwidth $\sigma=0.8 \mathrm{meV}$ ) for excitation powers between 0.12 and $0.58 \mu \mathrm{W}$. Solid lines: Bloch equation model. (b) Variation of the signal magnitude $\Delta R_{m}\left(\omega_{Q D}\right) / R_{0}$ with pump power. (c) Phase shift of the QD polarization vs. pump power.

The dispersive $\Delta R$ line shape observed in Fig. 9(a) for below band gap excitation and small time delays is the signature of the OSE in the weak excitation limit (59). It reflects a transient light-induced blue shift QD exciton resonance. In the presence of an AC electric field of frequency $\omega_{p}$, the transition frequency of a two-level system shifts by $\Delta \omega_{0}(t)=\sqrt{\left[\left(\omega_{0}-\omega_{p}\right)^{2}+\Omega_{R}(t)^{2}\right]}+\omega_{p}-\omega_{0}$. Here, $\omega_{0}$ is the transition frequency without external field, $\Omega_{R}(t)=\mu \cdot E_{p}(t) / \hbar$ is the Rabi frequency, $\mu$ the transition dipole moment and $E_{p}(t) \cdot \cos \left(\omega_{p} t\right)$ the (pump) AC electric field. The blue shift $\Delta \omega_{0}(t)$ of the QD absorption resonance results in a dispersive $\Delta R(\omega) / R_{0}$ line shape, which can be approximated as $\Delta R(\omega) / R_{0} \propto \Delta \omega_{0, \max } \cdot \partial \alpha(\omega) / \partial \omega$, where $\alpha(\omega)$ is the QD absorption spectrum and $\Delta \omega_{0, \max }$ is the maximum blue shift. Thus, in the weak excitation limit, the amplitude of the $\Delta R(\omega) / R$ signal is expected to increase linearly with increasing pump power, without change of the line shape. The spectra of Fig. 10(a) taken with pump powers $\leq 0.2 \mu \mathrm{W}$ exactly display this behavior. For such pump powers, the Rabi frequency has a maximum value of $\Omega_{R, \max }=1.75 \mathrm{meV} \simeq 5 \Delta \omega_{0, \max }$.

The origin of this transient blue shift becomes clear from an analysis of the optical Bloch equations. We describe the QD as a two-level system with a radiative lifetime of $T_{1}=100 \mathrm{ps}$ corresponding to a dipole moment $\mu=50$ Debye $(45 ; 34 ; 46)$. A dephasing time of $T_{2}=8 \mathrm{ps}$ is assumed to account for our finite monochromator resolution. It is important to stress that since we know both power and duration $\tau_{p}$ of the pump pulses and the spatial resolution of about $250 \mathrm{~nm}$, the electric field of the pump laser is estimated to within a factor of 2 and no free parameters enter the simulation.

The calculated dynamics of the QD polarization in the weak excitation limit are displayed in the rotating frame in Fig. 12(a). The probe field resonant to the exciton line changes the QD population and drives a coherent polarization oscillating at the QD resonance frequency $\omega_{Q D}$. This polarization is $90^{\circ}$ phase-shifted with respect to the probe field $\left(\operatorname{Re}\left[P_{Q D}\right]=0\right)$. During the presence of the pump pulse, the polarization is externally driven, leading to oscillations at the detuning frequency $\omega_{\text {det }}=\omega_{0}-\omega_{p}$. After the interaction, the polarization is phase-shifted by $\Delta \phi \approx \int \Delta \omega_{0}(t) d t$. It is this shift $\Delta \phi$ of the QD polarization which changes the product $E_{p r}(\omega) \cdot E_{Q D}(\omega)$ of the complex electric fields and therefore the line shape. Fourier-transformation of the polarization dynamics gives directly the dispersive line shape of the $\Delta R(\omega)$ spectrum in the weak excitation limit, $\Delta \phi<40^{\circ}$, at early delay times (Fig. 9, inset in Fig. 12(a)). The simulation also reproduces the timedependent data shown in Fig. 11. Evidently, a vanishing nonlinearity at positive time delays is predicted by the Bloch model, since then the pump laser interacts with the sample before the excitonic polarization is created. At 

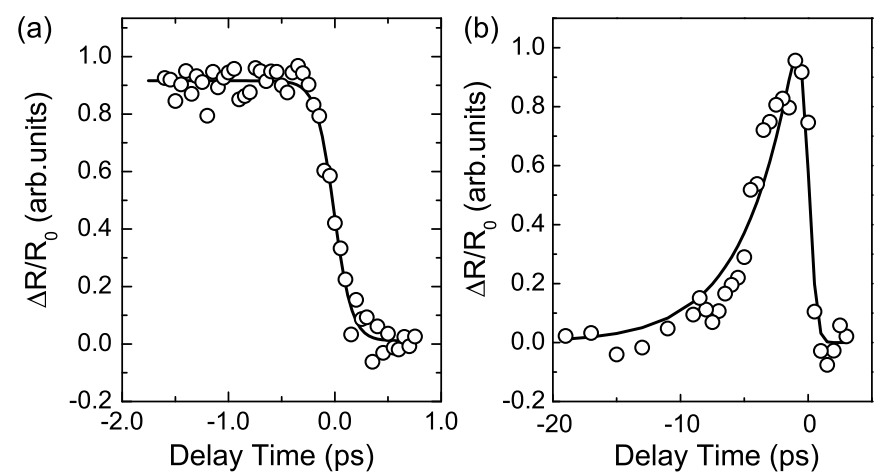

Figure 11. Time evolution of $\Delta R_{m}(\Delta t) / R_{0}$ for a single QD at $\omega_{Q D}=1.6544 \mathrm{eV}$. Here 200-fs pump pulses with a power of $58 \mu \mathrm{W}$ were centered at $1.640 \mathrm{eV} . \Delta R_{m}(\Delta t>0)$ vanishes and the signal for $\Delta t<0$ decays on a ps time scale. (a) 3 ps time scale. (b) 25 ps time scale.

negative delays, the OSE nonlinearity is expected to decay with the dephasing time of the polarization. The fact that we reproduce both predictions of this simple model experimentally is quite striking. In particular, we find within our signal-to-noise ratio, no measurable nonlinearity at $\Delta t>0$. This indicates that we are indeed probing a pure light-induced shift of the resonance and that nonlinearities induced by real carriers generated by one- or two-photon absorption in the surrounding of the QD obviously play a negligible role (40). This conclusion is strongly supported by recording transient nonlinear spectra at different negative delay times between 0 and -10 ps. Here, pronounced spectral oscillations are observed which are quantitatively fit by the Bloch equation. Thus, even under femtosecond excitation, the nonlinear response of the IQD for below band gap excitation is very close to that of an isolated atomic system and it appears that the excitonic QD excitation is only very weakly influenced by the complex solid state environment. To be precise, one should note that from our experiments one cannot directly tell whether the 8 ps decay at negative delay times reflects the polarization dephasing time. We are spectrally resolving the QD nonlinearity with a monochromator with about $100 \mu \mathrm{eV}$ resolution and this finite resolution puts an upper limit of slightly less than $10 \mathrm{ps}$ to the measurable decay. Thus the 8 ps decay is close to our instrument resolution and only gives a lower limit for the excitonic decoherence rate.
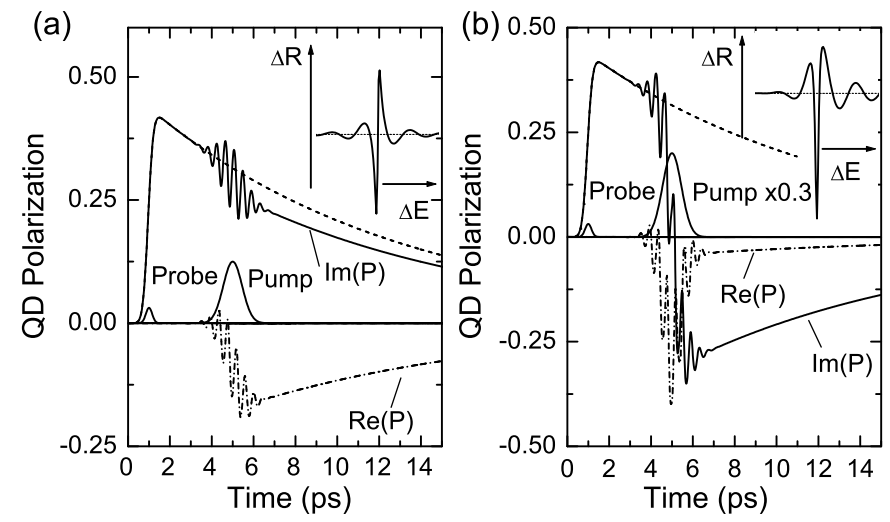

Figure 12. Bloch equation simulation of the single QD optical Stark effect. Shown is the time-dependent QD polarization $P_{Q D}$ in the rotating frame with (solid line) and without (dashed line) pump laser. Nonlinear $\Delta R$ spectra are given in the inset. (a) Weak excitation limit. (b) Strong excitation limit. The chosen pump power corresponds to a phase shift $\Delta \phi=172^{\circ}$.

For higher electric fields of the pump pulse, the weak excitation limit of the OSE nonlinearity is no longer valid. Experimentally one finds additional features in the transient reflectivity spectra [Fig. 10(a), traces for pump intensities of 0.32 and $0.58 \mu \mathrm{W}]$. These spectral oscillations are a direct consequence of the interaction of the QD 
polarization with the strong pump field. The pump laser induces pronounced large amplitude oscillations of QD polarization at the detuning frequency during the presence of the pump laser. This is illustrated in Fig. 12(b) showing the solution of Bloch equations for strong excitation with $\Omega_{R}=6 \mathrm{meV}\left(\omega_{\text {det }}=-10 \mathrm{meV}\right)$. A large phase shift $\Delta \phi$ of $172^{\circ}$ of the QD polarization results from this interaction and the nonlinear $\Delta R$ spectrum shows additional oscillatory structures on the high energy side, as found in the experiment. This large amplitude phase rotation corresponds to the observation of gain on the resonance of a single QD. In the Bloch sphere representation this phase rotation basically reflects a nutation-like motion of the Bloch vector, resulting in change in azimuthal angle after the interaction. A comparison between experimental spectra and simulation [solid lines in Fig. 10(a)] allows us to quantify the phase shift $\Delta \phi$ experienced by the QD polarization. In Fig. 10(c) we plot $\Delta \phi$ obtained from the simulation of the data in Fig. 10(a) as a function of the pump power $P_{p u}$. We find a linear increase in $\Delta \phi$ with $P_{p}$. This means that the light shift also increases linearly in our experiment, despite the saturation of $\Delta R_{m}$. This linear increase in the polarization phase $\Delta \phi$ is somewhat analogous to the pulse area theorem for Rabi oscillations of the population of a two-level system when driven with a resonant pulse. Currently, we can quantitatively measure the phase shift with an accuracy of about $10^{\circ}$ and achieve phase rotations of as much as $200^{\circ}$. Control of the exciton density, on the other hand, has been established above by the observation of Rabi oscillations when varying the pulse area of a resonant excitation pulse. The result show that a sequence of a resonant and an off-resonant laser pulse gives full control over both amplitude and phase of the coherent excitonic polarization. In particular, we can switch the QD from absorption to gain within about 1 ps.

\section{COUPLING TWO QUANTUM DOTS VIA THE DIPOLE-DIPOLE INTERACTION}

Coherent control of excitonic transitions in single quantum dots, as demonstrated in the last section, is an essential prerequisite for exploring excitonic couplings between adjacent dots and attempting to implement potetially scalable two-qubit operations. During the last years, different microscopic coupling schemes have been proposed theoretically for achieving such implementations, among them coupling via photonic or plasmonic nanoresonator modes, via optical phonon wavepackets or through dipolar interactions. In particular, ultrafast optical realizations of two-qubit operations in dipole-coupled QDs have been studied theoretically in some detail $(67 ; 22 ; 23 ; 68 ; 69)$.

Experimental studies of the proposed ideas have so far not been reported. This is partly due to a lack of suitable experimental methods. Since the strength of the dipole-dipole interaction depends strongly on both the geometric arrangment, (orientation and separation of the dipoles), and on the microscopic interaction (permanent dipole couplings, van der Waals dispersion forces, Förster dipole energy transfer, ...), studies of single nanosystems and/or ordered and homogeneous nanoarrays are often needed to resolve such copulings. Such experiments are scarce and have so far investigated a pair of molecules in an organic crystal (70) or the light-harvesting-2 complex (71) with steady-state techniques. Here, we demonstrate that combining high spatial resolution with time-resolving optical techniques allows for a separation of different couplings through their individual real-time dynamics and for controlling nanosystems on ultrashort time scales (39).

To probe dipole interactions between two individual QDs, we go back to the experimental situation depicted in Fig. 7. In Sec. 4.2 we have discussed experiments demonstrating coherent population control of the QD resonance at energy of $1.652 \mathrm{eV}$ (QD A). In these experiments, we have probed the pump-induced biexcitonic nonlinearity of this QD. The broad spectral bandwidth of the femtosecond probe pulses enables us to simultaneously probe also the pump-induced nonlinear optical response of the other quantum dots detected at this NSOM tip position. We focus on the optical nonlinearity of the neighboring QD resonance at $1.649 \mathrm{eV}$ (QD B) and study now the effect of a single-exciton excitation of QD A on the optical nonlinearity of this QD. Nonlinear spectra $\Delta R_{B}$ of QD B recorded with resonant excitation of QD A are displayed in Fig. 13(a). The excitation conditions are identical to those in Fig. 7(c) with an excitation pulse area of $\theta=0.75 \pi$.

Now, optical nonlinearities are observed both at positive and negative $\Delta t$, the latter being evident from the non-instantaneous rise of the signal in Fig. 13(b). In contrast to the absorptive line shape in Fig. 7(b), the nonlinear spectra display a time-independent dispersive line shape, reflecting a transient blue shift of the exciton resonance which does not change much with time delay. From the amplitude and shape of the nonlinear spectra we deduce a line shift of $30 \pm 15 \mu \mathrm{eV}$ around zero time delay. As seen in Fig. 13(b), the time evolution of $\Delta R_{B, m}$, defined as the difference between maximum and minimum of $\Delta R_{B}(\omega)$, is very different from that observed at 

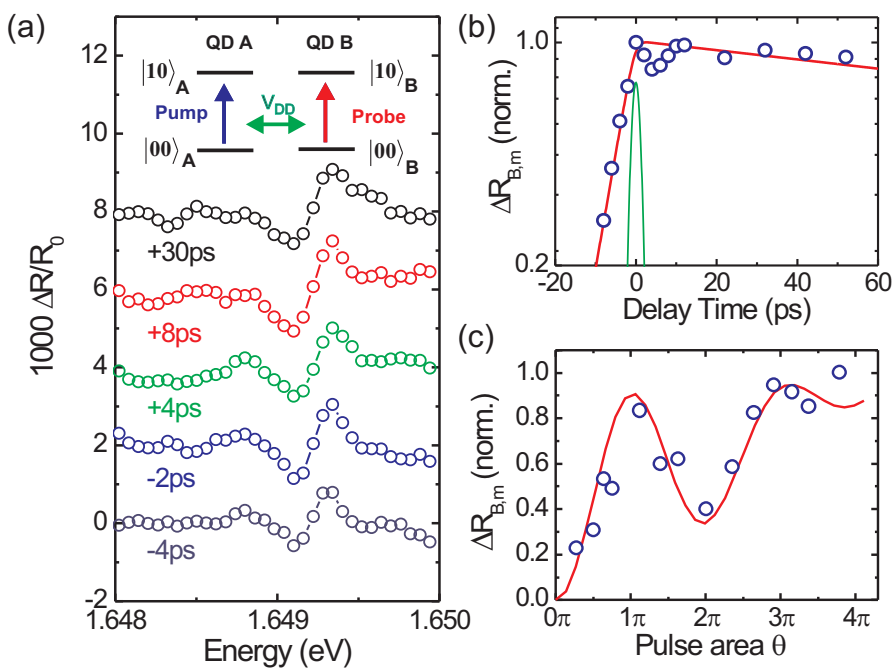

Figure 13. (a) Nonlinear $\Delta R$ spectra of quantum dot $\mathrm{B}$ for resonant single-exciton excitation of $\mathrm{QD} \mathrm{A}$ at $1.652 \mathrm{eV}$ as a function of time delay $\Delta t$. The pulse area of the 2-ps excitation pulses is $\theta \simeq 0.75 \pi$. Inset: Excitonic $|00\rangle \rightarrow|10\rangle$ transitions in QD A and QD B coupled through $V_{D D}$. (b) Time dynamics of $\Delta R_{B, m}(\Delta t)$. The excitation conditions are the same as in (a) and the time resolution of the experiment is indicated (thin solid line). (c) Rabi oscillation in a coupled QD. Magnitude of $\Delta R_{B, m}(\Delta t=10 \mathrm{ps})$ as a function of the field amplitude of the pump laser. The solid line shows a simulation based on an optical Bloch equation model.

the biexciton resonance. At negative time delays $\Delta R_{B, m}(\Delta t)$ shows a rise with a time constant of about 6 ps, followed by a slight dip and a slower decay on a time scale of more than $100 \mathrm{ps}$. The change of $\Delta R_{B, m}$ with the excitation field displays clear Rabi oscillations, in phase with those of Fig. 6(d).

To discuss these results, we stress the following observations: (i) Dispersive line shapes, caused by a transient blue shift of the QD resonance, are observed at all time delays and we find no signature of absorptive $\Delta R$ changes which would reflect pump-induced changes of the exciton population of QD B. This indicates that the observed nonlinearity is not due to an exciton relaxation between QD A and B. (ii) The presence of a strong laser field gives rise to transient excitonic line shifts via the optical Stark effect (OSE). However, as shown in Sec. 4.2, the OSE leads to optical nonlinearities at negative time delays $(\Delta t<0)$ only. Also, for a pump frequency above the exciton resonance, a red-shift of the QD line is expected, in contradiction with our present findings. (iii) There is a clear correlation between the pulse-area dependence of $\Delta R_{X X}$ in Fig. 8(d) and of $\Delta R_{B}$ in Fig. 13(c).

The data in Fig. 13 thus reflect an electronic coupling between the QDs A and B. The most likely candidate for such an interaction is a dipole-dipole coupling between both QDs. Theoretical studies $(22 ; 23 ; 67 ; 68)$ indicate that two different mechanisms can contribute: resonant Förster energy transfer and direct Coulomb interaction between permanent excitonic dipole moments. For two quantum dots separated by less than the wavelength of light, pulsed optical excitation of one QD leads to the re-emission of a transient electric field which can be reabsorbed by the second QD, thus (Förster) transferring the excitation. The interaction Hamiltonian $H_{F}=V_{F} p_{A} p_{B}^{*}+c . c$ includes the coupling $V_{F} \propto \mu_{A} \mu_{B} / R_{A B}^{3}$ between coherent excitonic polarizations $p_{i}(t)=$ $|10\rangle_{i}\left\langle\left. 01\right|_{i}+\right.$ c.c. in QDs A and B. The coupling strength is determined by the transition dipole moments $\mu_{i}=$ $\left|\left\langle 00\left|\vec{M}_{i}\right| 10\right\rangle_{i}\right|\left(\vec{M}_{i}\right.$ : dipole operator) and the QD separation $R_{A B}$. In the strong coupling limit, $V_{F} / \hbar$ is larger than the detuning $\Delta \omega=\omega_{A}-\omega_{B}$ between the QD resonances and the dephasing rate $1 / T_{2}$, leading to entangled states of the coupled system and cooperative effects in its radiative decay $(72 ; 70 ; 68)$. In the weak coupling limit, $V_{F} \ll \hbar \Delta \omega, \hbar / T_{2}$, the interaction induces a population relaxation between the coupled states (73).

The direct dipole interaction $H_{D}$ on the other hand involves permanent excitonic dipole moments and thus interaction between the exciton populations $n_{i}=|10\rangle_{i}\left\langle\left. 10\right|_{i}\right.$ with $H_{D}=V_{D} n_{A} n_{B}$ and $V_{D} \propto d_{A} d_{B} / R_{A B}^{3}$. Here, $d_{i}$ represents the permanent dipole moment originating from a shift of the electron and hole charge distributions in the exciton. This interaction leads to a biexcitonic energy shift $V_{D}$ in case that both QDs are excited (22). 

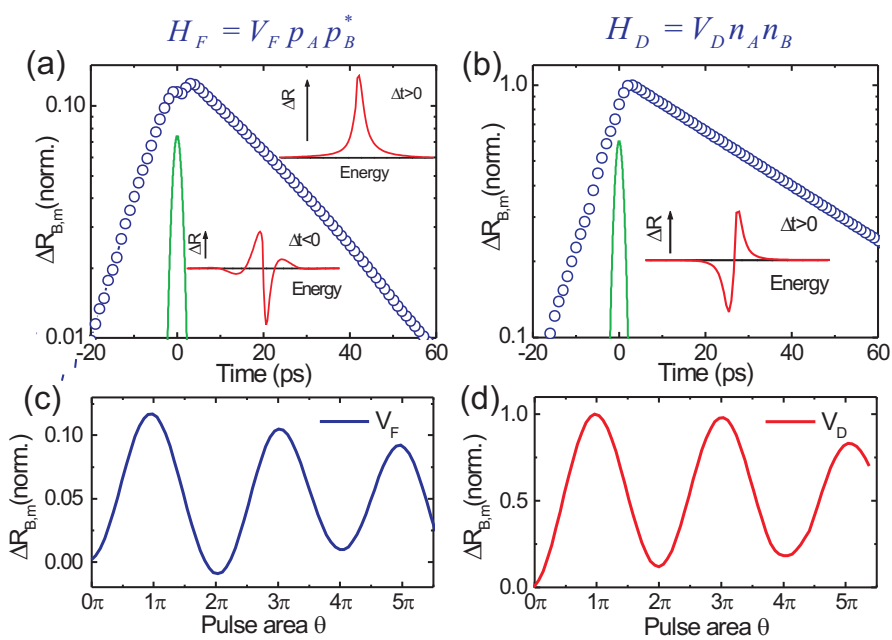

Figure 14. (a) Simulation of optical nonlinearities of two QD coupled by Förster energy transfer for excitation conditions similar to Fig. 13. The nonlinear spectra (inset) display an absorptive line shape at $\Delta t>0$ and dispersive red-shifted lineshape at $\Delta t<0$. (b) Coupling via permanent excitonic dipole moments. For $V_{D}>0$, the nonlinear spectra (inset) reflect a blue shift of the exciton line at all time delays. Pump-induced Rabi oscillations $(\Delta t=10 \mathrm{ps})$ for Förster (c) and for direct dipole coupling (d).

To examine these two interaction mechanisms, nonlinear optical spectra are calculated from the time evolution of the density matrix in rotating wave approximation. Here, the QDs are treated as effective two-level systems (states $|00\rangle_{i},|10\rangle_{i}$ ), interacting with the pump and probe fields and coupled via the dipole-dipole interaction. Most of the parameters of these calculations such as $\omega_{i}, \mu_{i}, T_{2, i}$, and electric field profiles of the lasers are quantitatively known. The basic unknown is the mechanism and strength of the dipole-dipole interaction.

For the Förster mechanism, the time-evolution of the spectra depends critically on the ratios of $V_{F}, \hbar \Delta \omega$, and $\hbar / T_{2}$. In our case, typical interdot distances are limited by the finite exciton size to about $20 \mathrm{~nm}$, giving $V_{F} \simeq 30 \mu \mathrm{eV}$ for $\mu=60 \mathrm{D}$. Therefore $V_{F} \leq \hbar / T_{2}(0.1 \mathrm{meV}) \ll \hbar \Delta \omega(3 \mathrm{meV})$, i.e., we are in the weak coupling limit. At negative delay times $\Delta t<0, \Delta R_{B, m}$ is due to the optical Stark effect induced by the pump field with a dispersive lineshape reflecting a red shift of the exciton line, and a rise of $\Delta R_{B, m}(\Delta t<0)$ with $T_{2, B}$ (Fig. 14(a)). At $\Delta t>0$, the Förster mechanism induces exciton population relaxation between both QDs, resulting in absorptive line shapes. The decay of $\Delta R_{B, m}(\Delta t>0)$ reflects both the exciton lifetime $T_{1, A} \simeq 40 \mathrm{ps}$ and the exciton transfer rate which scales as $\Gamma_{F} \propto V_{F}^{2} T_{2}\left[1+\left(\Delta \omega T_{2}\right)^{2}\right]^{-1}$ (73). Although the excitation field dependence of $\Delta R_{B, m}(\Delta t=10 \mathrm{ps})$ (Fig. 14(c)) displays Rabi oscillations, the line shapes and the the temporal dynamics of $\Delta R_{B, m}$ are in disagreement with the experiment. Also the amplitude of $\Delta R_{B, m}$ is much smaller than in the experiment. We infer that dipole coupling via the Förster mechanism is of minor importance for our QDs.

For a direct dipole interaction $H_{D}$ between permanent excitonic dipole moments, excitation of QD A transiently shifts the energy of QD B by $V_{D}$. The sign of this shift depends on the sign of $V_{D}$ and - thus - a blue shift occurs for parallel dipoles $d_{A}$ and $d_{B}$. For a shift smaller than the homogeneous exciton linewidth, the coupling results in a dispersive shape of $\Delta R$ (Fig. 14(b)). Both direct dipole coupling and OSE contribute to the line shifts at $\Delta t<0$ and net blue shifts are observed if the Coulomb coupling is stronger than the OSE. The signal at $\Delta t<0$ rises with $T_{2, B}$. For $\Delta t>0, \Delta R_{B, m}$ decays exponentially with the exciton lifetime $T_{1, A}$, as there is no population transfer between the dots. The amplitude of $\Delta R_{B, m}$ monitors the exciton population in QD A and the intensity dependence of the pump-induced Rabi oscillation (Fig. 14(b)) is thus similar to that found in the single exciton manipulation experiments.

The experimental line shapes and Rabi oscillations are in good agreement with the direct coupling model. The calculated decay of $\Delta R_{B, m}$, however, is faster. This discrepancy may reflect signal contributions from more delocalized excitonic transitions in the environment of QD A (42). Such states have smaller dipole moments and thus longer radiative lifetimes. Their presence may also lead to finite dipole shifts that persist on time scales 
longer than $T_{1, A}$. This notion is supported by finding experimentally a finite optical nonlinearity from QD B when the excitation pulse is slightly detuned from the resonance of QD A. For such a nonresonant excitation, however, Rabi oscillations are not observed. This indicates that the direct coupling between permanent excitonic dipole moments is the dominant interaction mechanism. Apart from the permanent dipoles, the Coulomb interaction between excitons in QD A and B may lead to induced charge rearrangements which lower the energy (formation of distant biexcitons). The absence of a redshift in the experiment points to a dominance of dipole repulsion over such correlation effects.

It is interesting to ask whether the weak Förster coupling is a general property of this class of QD samples. The energy statistics of the localized exciton states are heavily influenced by level repulsion effects (42), resulting in finite energy splittings between excitons in neighboring QDs. Such splittings are typically $1-3 \mathrm{meV}$ and thus stronger than the dipole coupling. Thus it is quite unlikely to find near-resonance situations between adjacent QDs and Förster coupling is expected to be weak in general.

\section{SUMMARY AND CONCLUSIONS}

In summary, we have introduced a novel technique, ultrafast near-field optical spectroscopy, to probe the nonlinear optical response from single semiconductor quantum dots. We have used this technique to demonstrate coherent control over amplitude and phase of the excitonic QD polarization. Rabi oscillations of up to $4 \pi$ are induced and probed by ultra-fast light pulses. It appears that even in relatively weakly confined interface quantum dots, the ultrafast polarization dynamics are in many respects similar to those of an atomic system, yet with an enhanced dipole moment. Only when interacting with strong excitation pulses with an area of order $2 \pi$, excitation-induced dephasing due to Coulomb-mediated many-body interactions is limiting the visibility of Rabi oscillations. One may expect that using quantum dots with larger confinement energies may reduce excitation-induced dephasing. Yet, so far the experiments on Rabi oscillations in more strongly confined self-assembled quantum dots seem to indicate that here other factors, such as enhanced exciton-phonon coupling, may be important additional decoherence sources. Certainly, the microscopic origin of exciton decoherence in single quantum dots will be the topic of much additional experimental and theoretical work in the near future.

The introduced experimental technique probes transient optical nonlinearities in a broad spectral range and thus is particularly well suited to study excitonic couplings. This allowed us to demonstrate coupling between permanent excitonic dipole moments in a pair of adjacent quantum dots. The coupling strength of about $30 \mu \mathrm{eV}$ is still about one order of magnitude too small to implement a nonlocal conditional quantum gate as proposed in (22). An increase in coupling should readily be achievable by applying moderate lateral electric fields and two-qubit gating times of few ps seem feasible (23). Recent progress in nano-fabrication allows for manufacturing linear arrays of vertically and laterally stacked quantum dots with well defined interdot distances. Such systems may permit to go beyond two-qubit operations towards scalable qubit arrays, even though statistical variations of the coupling parameters within such arrays and excitation-induced decoherence still pose major technological challenges. Either energy-selective addressing (with inherently limited scalability) or cellular-automaton schemes with globally applied multicolor pulse sequences may be used for encoding information in such arrays. The now established real-time probing of many-body interactions between individual solid-state nanostructures will certainly be of key importance for future progress in this area.

\section{ACKNOWLEDGMENT}

High-quality semiconductor samples for this work have been provided by Soheyla Eshlaghi and Andreas D. Wieck (Ruhr-Universität Bochum). We are very grateful for theoretical support by and stimulating discussion with Markus Glaneman, Vollrath Martin Axt and Tilmann Kuhn (Universität Münster), Andreas Knorr (Technische Universität Berlin), and Roland Zimmermann (Humboldt-Universität Berlin). Financial support by the Deutsche Forschungsgemeinschaft (SFB296) and the European Union through the SQID program is gratefully acknowledged. 


\section{References}

[1] M. A. Nielsen, , and I. L. Chuang, Quantum Computation and Quantum Information, Cambridge University Press, Cambridge, 2000.

[2] N. Gershenfeld and I. L. Chuang, "Bulk Spin-Resonance Quantum Computation," Science 275, pp. 350-356, 1997.

[3] L. M. K. Vandersypen, M. Steffen, G. Breyta, C. S. Yannoni, M. H. Sherwood, and I. L. Chuang, "Experimental realization of Shor's quantum factoring algorithm using nuclear magnetic resonance," Nature 414, pp. 883-887, 2001.

[4] J. C. Cirac and P. Zoller, "Quantum Computations with Cold Trapped Ions," Phys. Rev. Lett. 74, pp. 40914094, 1995.

[5] A. Sorensen and K. Molmer, "Quantum Computation with Ions in Thermal Motion," Phys. Rev. Lett. 82, pp. 1971-1974, 1999.

[6] S. Gulde, M. Riebe, G. P. T. Lancaster, C. Becher, J. Eschner, H. Häffner, F. Schmidt-Kaler, I. L. Chuang, and R. Blatt, "Implementation of the Deutsch-Jozsa algorithm on an ion-trap quantum computer," Nature 421, pp. 48-50, 2003.

[7] F. Schmidt-Kaler, H. Häffner, M. Riebe, S. Gulde, G. P. T. Lancaster, T. Deuschle, C. Becher, C. F. Roos, J. Eschner, and R. Blatt, "Realization of the Cirac-Zoller controlled-NOT quantum gate," Nature 422, pp. 408-411, 2003.

[8] Q. A. Turchette, C. J. Hood, W. Lange, H. Mabuchi, and H. J. Kimble, "Measurement of Conditional Phase Shifts for Quantum Logic," Phys. Rev. Lett. 75, pp. 4710-4713, 1995.

[9] G. K. Brennen, C. M. Caves, and I. H. Deutsch, "Quantum Logic Gates in Optical Lattices," Phys. Rev. Lett. 82, pp. 1060-1063, 1999.

[10] M. Woldeyohannes and S. John, "Coherent control of spontaneous emission near a photonic band edge: A qubit for quantum computation," Phys. Rev. A 60, pp. 5046-5068, 1999.

[11] J. E. Mooij, T. P. Orlando, L. Levitov, L. Tian, C. H. van der Wal, and S. Lloyd, "Josephson PersistentCurrent Qubit," Science 285, pp. 1036-1039, 1999.

[12] T. Yamamoto, Y. A. Pashkin, O. Astafiev, Y. Nakamura, and J. S. Tsai, "Demonstration of conditional gate operation using superconducting charge qubits," Nature 425, pp. 941-944, 2003.

[13] J. D. Franson, "Cooperative Enhancement of Optical Quantum Gates," Phys. Rev. Lett. 78, pp. 3852-3855, 1997.

[14] E. Knill, R. Laflamme, and G. J. Milburn, "A scheme for efficient quantum computation with linear optics," Nature 409, pp. 46-52, 2001.

[15] A. Zrenner, "A close look on single quantum dots," J. Chem. Phys. 112, p. 7790, 2000.

[16] K. Brunner, G. Abstreiter, G. Böhm, G. Tränkle, , and G. Weimann, "Sharp-Line Photoluminescence and Two-Photon Absorption of Zero-Dimensional Biexcitons in a GaAs/AlGaAs Structure," Phys. Rev. Lett. 73, pp. 1138-1141, 1994.

[17] H. F. Hess, E. Betzig, T. D. Harris, L. N. Pfeiffer, and K. W. West, "Near-field spectroscopy of the quantum constituents of a luminescent system," Science 264, pp. 1740-1745, 1994.

[18] D. Gammon, E. S. Snow, B. V. Shanabrook, D. S. Katzer, , and D. Park, "Fine Structure Splitting in the Optical Spectra of Single GaAs Quantum Dots," Phys. Rev. Lett. 76, pp. 3005-3008, 1996. 
[19] P. Hawrylak, G. A. Narvaez, M. Bayer, and A. Forchel, "Excitonic Absorption in a Quantum Dot," Phys. Rev. Lett. 85, pp. 389-392, 2000.

[20] P. Borri, W. Langbein, S. Schneider, U. Woggon, R. L. Sellin, D. Ouyang, and D. Bimberg, "Ultralong Dephasing Time in InGaAs Quantum Dots," Phys. Rev. Lett. 87, p. 157401, 2001.

[21] M. Bayer and A. Forchel, "Temperature dependence of the exciton homogeneous linewidth in In0.60Ga0.40As/GaAs self-assembled quantum dots," Phys. Rev. B 65, p. 041308, 2002.

[22] E. Biolatti, R. Iotti, P. Zanardi, and F. Rossi, "Quantum Information Processing with Semiconductor Macroatoms," Phys. Rev. Lett. 85, pp. 5647-5650, 2000.

[23] E. Biolatti, I. D'Amico, P. Zanardi, and F. Rossi, "Electro-optical properties of semiconductor quantum dots: Application to quantum information processing," Phys. Rev. B 65, p. 075306, 2002.

[24] P. Chen, C. Piermarocchi, and L. J. Sham, "Control of Exciton Dynamics in Nanodots for Quantum Operations," Phys. Rev. Lett. 87, p. 067401, 2001.

[25] C. Piermarocchi, P. Chen, Y. S. Dale, and L. J. Sham, "Theory of fast quantum control of exciton dynamics in semiconductor quantum dots," Phys. Rev. B 65, p. 075307, 2002.

[26] N. H. Bonadeo, G. Chen, D. Gammon, D. S. Katzer, D. Park, and D. G. Steel, "Nonlinear Nano-Optics: Probing One Exciton at a Time," Phys. Rev. Lett. 81, pp. 2759-2762, 1998.

[27] Y. Toda, T. Sugimoto, M. Nishioka, and Y. Arakawa, "Near-field coherent excitation spectroscopy of InGaAs/GaAs self-assembled quantum dots," Appl. Phys. Lett. 76, pp. 3887-3889, 2000.

[28] T. Flissikowski, A. Hundt, M. Lowisch, M. Rabe, and F. Henneberger, "Photon Beats from a Single Semiconductor Quantum Dot," Phys. Rev. Lett. 86, pp. 3172-3175, 2001.

[29] T. H. Stievater, X. Li, D. G. Steel, D. Gammon, D. S. Katzer, D. Park, C. Piermarocchi, , and L. J. Sham, "Rabi Oscillations of Excitons in Single Quantum Dots," Phys. Rev. Lett. 87, p. 133603, 2001.

[30] H. Kamada, H. Gotoh, J. Temmyo, T. Takagahara, and H. Ando, "Exciton Rabi Oscillation in a Single Quantum Dot," Phys. Rev. Lett. 87, p. 246401, 2001.

[31] H. Htoon, T. Takagahara, D. Kulik, O. Baklenov, A. L. Holmes, Jr., and C. K. Shih, "Interplay of Rabi Oscillations and Quantum Interference in Semiconductor Quantum Dots," Phys. Rev. Lett. 88, p. 087401, 2002.

[32] A. Zrenner, E. Beham, S. Stuffer, F. Findeis, M. Bichler, and G. Abstreiter, "Coherent properties of a two-level system based on a quantum-dot photodiode," Nature 418, pp. 612-614, 2002.

[33] L. Besombes, J. J. Baumberg, , and J. Motohisa, "Coherent Spectroscopy of Optically Gated Charged Single InGaAs Quantum Dots," Phys. Rev. Lett. 90, p. 257402, 2003.

[34] T. Guenther, C. Lienau, T. Elsaesser, M. Glanemann, V. M. Axt, T. Kuhn, S. Eshlaghi, , and A. D. Wieck, "Erratum: Coherent Nonlinear Optical Response of Single Quantum Dots Studied by Ultrafast Near-Field Spectroscopy [Phys. Rev. Lett. 89, 057401 (2002)]," Phys. Rev. Lett. 89, p. 179901, 2002.

[35] A. Vagov, V. M. Axt, and T. Kuhn, "Electron-phonon dynamics in optically excited quantum dots: Exact solution for multiple ultrashort laser pulses," Phys. Rev. B 66, p. 165312, 2002.

[36] J. Förstner, C. Weber, J. Danckwerts, and A. Knorr, "Phonon-Assisted Damping of Rabi Oscillations in Semiconductor Quantum Dots," Phys. Rev. Lett. 91, p. 127401, 2003.

[37] X. Li, Y. Wu, D. Steel, D. Gammon, T. H. Stievater, D. S. Katzer, D. Park, C. Piermarocchi, , and L. J. Sham, "An All-Optical Quantum Gate in a Semiconductor Quantum Dot," Science 301, pp. 809-811, 2003. 
[38] T. Guenther, C. Lienau, T. Elsaesser, M. Glanemann, V. M. Axt, , and T. Kuhn, "Guenther et al. Reply:," Phys. Rev. Lett. 90, p. 139702, 2003.

[39] T. Unold, K. Mueller, C. Lienau, T. Elsaesser, and A. D. Wieck, "Optical Control of Excitons in a Pair of Quantum Dots Coupled by the Dipole-Dipole Interaction," Phys. Rev. Lett. 94, p. 137404, 2005.

[40] T. Unold, K. Mueller, T. E. C. Lienau, and A. D. Wieck, "Optical Stark Effect in a Quantum Dot: Ultrafast Control of Single Exciton Polarizations," Phys. Rev. Lett. 92, p. 157401, 2004.

[41] F. Intonti, V. Emiliani, C. Lienau, T. Elsaesser, R. Nötzel, , and K. H. Ploog, "Near-field optical spectroscopy of localized and delocalized excitons in a single GaAs quantum wire," Phys. Rev. B 63, pp. 075313, 1-5, 2001.

[42] F. Intonti, V. Emiliani, C. Lienau, T. Elsaesser, V. Savona, E. Runge, R. Zimmermann, R. Nötzel, , and K. H. Ploog, "Quantum Mechanical Repulsion of Exciton Levels in a Disordered Quantum Well," Phys. Rev. Lett. 87, pp. 076801, 1-4, 2001.

[43] V. Emiliani, F. Intonti, C. Lienau, T. Elsaesser, R. Nötzel, and K. H. Ploog, "Near-field optical imaging and spectroscopy of a coupled quantum wire-dot structure," Phys. Rev. B 64, pp. 155316, 1-9, 2001.

[44] D. Gammon, E. S. Snow, B. V. Shanabook, D. S. Katzer, and D. Park, "Homogeneous liniwidths in the optical spectrum of a single gallium arsenide quantum dot," Science 273, pp. 87-90, 1996.

[45] J. R. Guest, T. H. Stievater, X. Li, J. Cheng, D. G. Steel, D. Gammon, D. S. Katzer, D. Park, C. Ell, A. Thränhardt, G. Khitrova, and H. M. Gibbs, "Measurement of optical absorption by a single quantum dot exciton," Phys. Rev. B 65, pp. 241310(R), 1-4, 2002.

[46] A. Thränhardt, C. Ell, G. Khitrova, and H. M. Gibbs, "Relation between dipole moment and radiative lifetime in interface quantum dots," Phys. Rev. B 65, p. 035327, 2002.

[47] F. Intonti, V. Emiliani, C. Lienau, T. Elsaesser, V. Savona, E. Runge, R. Zimmermann, R. Nötzel, and K. H. Ploog, "Quantum mechanical repulsion of exciton levels in a disordered quantum well," Phys. Rev. Lett. 87, p. 076801, 2001.

[48] P. Lambelet, A. Sayah, M. Pfeffer, C. Philipona, and F. Marquis-Weible, "Chemically etched fiber tips for near-field optical microscopy: a process for smoother tips.," Appl. Optics 37, pp. 7289-7292, 1998.

[49] L. C. Andreani, G. Panzarini, and J. M. Gerard, "Strong-coupling regime for quantum boxes in pillar microcavities: Theory," Phys. Rev. B 60, p. 13276, 1999.

[50] C. Lienau, F. Intonti, T. Guenther, T. Elsaesser, V. Savona, R. Zimmermann, , and E. Runge, "Near-field autocorrelation spectroscopy of disordered semiconductor quantum wells," Phys. Rev. B 69, p. 085302, 2004.

[51] V. Savona, E. Runge, R. Zimmermann, F. Intonti, V. Emiliani, C. Lienau, and T. Elsaesser, "Level Repulsion of Localized Excitons in Disordered Quantum Wells," phys. stat. sol. (a) 190, pp. 625-629, 2002.

[52] H. Haug and S. W. Koch, Quantum theory of the optical and electronic properties of semiconductors, World Scientific, Singapore, 2 ed., 1994.

[53] B. Fluegel, N. Peyghambarian, G. Olbright, M. Lindberg, S. W. Koch, M. Joffre, D. Hulin, A. Migus, and A. Antonetti, "Femtosecond Studies of Coherent Transients in Semiconductors," Phys. Rev. Lett. 59, pp. 2588-2591, 1987.

[54] J. P. Sokoloff, M. Joffre, B. Fluegel, D. Hulin, M. Lindberg, S. W. Koch, A. Migus, A. Antonetti, and N. Peyghambarian, "Transient oscillations in the vicinity of excitons and in the band of semiconductors," Phys. Rev. B 38, pp. 7615-7621, 1988.

[55] H. Wang, K. Ferrio, D. G. Steel, Y. Z. Hu, R. Binder, , and S. W. Koch, "Transient nonlinear optical response from excitation induced dephasing in GaAs," Phys. Rev. Lett. 71, pp. 1261-1264, 1993. 
[56] A. Bertoni, P. Bordone, R. Brunetti, C. Jacoboni, and S. Reggiani, "Quantum Logic Gates based on Coherent Electron Transport in Quantum Wires," Phys. Rev. Lett. 84, pp. 5912-5915, 2000.

[57] B. R. Mollow, "Stimulated Emission and Absorption near Resonance for Driven Systems," Phys. Rev. A 5, pp. 2217-2222, 1972.

[58] H. Häffner, S. Gulde, M. Riebe, G. Lancaster, C. Becher, J. Eschner, F. Schmidt-Kaler, and R. Blatt, "Precision Measurement and Compensation of Optical Stark Shifts for an Ion-Trap Quantum Processor," Phys. Rev. Lett. 90, p. 143602, 2003.

[59] A. Mysyrowicz, D. Hulin, A. Antonetti, A. Migus, W. T. Masselink, , and H. Morkoç, "” Dressed Excitons" in a Multiple-Quantum-Well Structure: Evidence for an Optical Stark Effect with Femtosecond Response Time," Phys. Rev. Lett. 56, pp. 2478-2751, 1986.

[60] W. H. Knox, D. S. Chemla, D. A. B. Miller, J. B. Stark, and S. Schmitt-Rink, "Femtosecond ac Stark effect in semiconductor quantum wells: Extreme low- and high-intensity limits," Phys. Rev. Lett. 62, pp. 1189-1192, 1989.

[61] C. Sieh, T. Meier, F. Jahnke, A. Knorr, S. W. Koch, P. Brick, M. Hübner, C. Ell, J. Prineas, G. Khitrova, and H. M. Gibbs, "Coulomb Memory Signatures in the Excitonic Optical Stark Effect," Phys. Rev. Lett. 82, pp. 3112-3115, 1999.

[62] M. Saba, F. Quochi, C. Ciuti, D. Martin, J.-L. Staehli, B. Deveaud, A. Mura, and G. Bongiovanni, "Direct observation of the excitonic ac Stark splitting in a quantum well," Phys. Rev. B 62, pp. R16322-R16325, 2000 .

[63] S. Schmitt-Rink and D. S. Chemla, "Collective Excitations and the Dynamical Stark Effect in a Coherently Driven Exciton System," Phys. Rev. Lett. 57, pp. 2752-2755, 1986.

[64] M. Combescot and R. Combescot, "Excitonic Stark Shift: A Coupling to "Semivirtual" Biexcitons," Phys. Rev. Lett. 61, pp. 117-120, 1988.

[65] S. Schmitt-Rink, D. S. Chemla, and H. Haug, "Nonequilibrium theory of the optical Stark effect and spectral hole burning in semiconductors," Phys. Rev. B 37, pp. 941-955, 1988.

[66] C. Ell, J. F. Müller, K. E. Sayed, and H. Haug, "Influence of Many-Body Interactions on the Excitonic Optical Stark Effect," Phys. Rev. Lett. 62, pp. 304-307, 1989.

[67] L. Quiroga and N. F. Johnson, "Entangled Bell and Greenberger-Horne-Zeilinger States of Excitons in Coupled Quantum Dots," Phys. Rev. Lett. 83, pp. 2270-2273, 1999.

[68] B. W. Lovett, J. H. Reina, A. Nazir, and G. A. D. Briggs, "Optical schemes for quantum computation in quantum dot molecules," Phys. Rev. B 68, p. 205319, 2003.

[69] A. Nazir, B. W. Lovett, S. D. Barrett, J. H. Reina, and G. A. D. Briggs, "Anticrossings in Förster coupled quantum dots," Phys. Rev. B 71, p. 045334, 2005.

[70] C. Hettich, C. Schmitt, J. Zitzmann, S. Kuhn, I. Gerhardt, and V. Sandoghdar, "Nanometer resolution and coherent optical dipole coupling of two individual molecules," Science 298, pp. 385-389, 2002.

[71] C. Hofmann, M. Ketelaars, M. Matsushita, H. Michel, T. J. Aartsma, and J. Köhler, "Single-Molecule Study of the Electronic Couplings in a Circular Array of Molecules: Light-Harvesting-2 Complex from Rhodospirillum Molischianum," Phys. Rev. Lett. 90, p. 013004, 2003.

[72] R. H. Dicke, "Coherence in Spontaneous Radiation Processes," Phys. Rev. 93, pp. 99-110, 1954.

[73] J. A. Leegwater, "Coherent versus Incoherent Energy Transfer and Trapping in Photosynthetic Antenna Complexes," J. Phys. Chem. 100, pp. 14403-14409, 1996. 